\title{
Timed Ang2-Targeted Therapy Identifies the Angiopoietin-Tie Pathway as Key Regulator of Fatal Lymphogenous Metastasis
}

Nicolas Gengenbacher ${ }^{1,2,3}$, Mahak Singhal ${ }^{1,2,3}$, Carolin Mogler ${ }^{4}$, Ling Hai ${ }^{5}$, Laura Milde ${ }^{1,2,3}$, Ashik Ahmed Abdul Pari ${ }^{1,2,3}$, Eva Besemfelder ${ }^{1}$, Claudine Fricke ${ }^{1}$, Daniel Baumann ${ }^{3,6}$, Stephanie Gehrs $1,2,3$, Jochen Utikal ${ }^{7,8}$, Moritz Felcht ${ }^{2,8}$, Junhao Hu${ }^{9}$, Matthias Schlesner ${ }^{5}$, Rienk Offringa ${ }^{6,10}$, Sudhakar R. Chintharlapalli ${ }^{11}$, and Hellmut G. Augustin ${ }^{1,2,12}$ 
ABSTRACT Recent clinical and preclinical advances have highlighted the existence of a previously hypothesized lymphogenous route of metastasis. However, due to a lack of suitable preclinical modeling tools, its contribution to long-term disease outcome and relevance for therapy remain controversial. Here, we established a genetically engineered mouse model (GEMM) fragment-based tumor model uniquely sustaining a functional network of intratumoral lymphatics that facilitates seeding of fatal peripheral metastases. Multiregimen survival studies and correlative patient data identified primary tumor-derived Angiopoietin-2 (Ang2) as a potent therapeutic target to restrict lymphogenous tumor cell dissemination. Mechanistically, tumor-associated lymphatic endothelial cells (EC), in contrast to blood vascular EC, were found to be critically addicted to the Angiopoietin-Tie pathway. Genetic manipulation experiments in combination with single-cell mapping revealed agonistically acting Ang2-Tie2 signaling as key regulator of lymphatic maintenance. Correspondingly, acute presurgical Ang2 neutralization was sufficient to prolong survival by regressing established intratumoral lymphatics, hence identifying a therapeutic regimen that warrants further clinical evaluation.

SIGNIFICANCE: Exploiting multiple mouse tumor models including a unique GEMM-derived allograft system in combination with preclinical therapy designs closely matching the human situation, this study provides fundamental insight into the biology of tumor-associated lymphatic EC and defines an innovative presurgical therapeutic window of migrastatic Ang2 neutralization to restrict lymphogenous metastasis.

\section{INTRODUCTION}

Since the advent of surgical oncology, metastasis has become the primary cause of cancer-related death (1). A plethora of research has covered the dissemination of tumor cells via the blood circulation (2). However, accumulating evi-

\footnotetext{
${ }^{1}$ Division of Vascular Oncology and Metastasis, German Cancer Research Center (DKFZ-ZMBH Alliance), Heidelberg, Germany. ${ }^{2}$ Department of Vascular Biology and Tumor Angiogenesis, European Center for Angioscience (ECAS), Medical Faculty Mannheim, Heidelberg University, Mannheim, Germany. ${ }^{3}$ Faculty of Biosciences, Heidelberg University, Mannheim, Germany. ${ }^{4}$ Institute of Pathology, TUM School of Medicine, Munich, Germany. ${ }^{5}$ Junior Group Bioinformatics and Omics Data Analytics, German Cancer Research Center (DKFZ), Heidelberg, Germany. ${ }^{6}$ Division of Molecular Oncology of Gastrointestinal Tumors, German Cancer Research Center (DKFZ), Heidelberg, Germany. ${ }^{7}$ Skin Cancer Unit, German Cancer Research Center (DKFZ), Heidelberg, Germany. ${ }^{8}$ Department of Dermatology, Venereology and Allergology, University Medical Center Mannheim, Heidelberg University, Mannheim, Germany. ${ }^{9}$ nterdisciplinary Research Center on Biology and Chemistry, Shanghai Institute of Organic Chemistry, Chinese Academy of Sciences, Shanghai, China. ${ }^{10}$ Department of Surgery, University Hospital Heidelberg, Heidelberg, Germany. ${ }^{11}$ Eli Lilly and Company, Indianapolis, Indiana. ${ }^{12}$ German Cancer Consortium, Heidelberg, Germany.
}

N. Gengenbacher and M. Singhal contributed equally as the first authors of this article.

Corresponding Author: Hellmut G. Augustin, European Center for Angioscience (ECAS), Medical Faculty Mannheim, Heidelberg University, and German Cancer Research Center (DKFZ-ZMBH Alliance), Im Neuenheimer Feld 280, 69120 Heidelberg, Germany. Phone: 49-6221-421500; Fax: 49-6221-421515; E-mail: augustin@angioscience.de dence from animal and clinical studies suggests that tumors can additionally exploit the lymphatic system for their spread to distant organ sites $(3,4)$. The importance of tumor lymphangiogenesis for metastatic progression has been demonstrated via diverse experimental strategies, including pharmacologic targeting of VEGFR3 signaling as well as genetic depletion of proliferating lymphatic endothelial cells (LEC; refs. 5, 6). Accordingly, intratumoral lymphatic vessel density, lymphovascular invasion, and metastatic growth in sentinel lymph nodes (LN) are all established biomarkers, predicting peripheral organ metastasis and poor survival in melanoma and many types of epithelial cancer (7-9). Phylogenetic mapping of patients with colorectal carcinoma revealed that at least a subset of distant metastases is more closely related to LN lesions than to primary tumors, hinting at a consecutive lymphogenous route of cancer cell seeding (10). Two recent animal studies indeed formally validated that LN metastases can serve as gateways for subsequent metastatic colonization of distant sites $(11,12)$. Yet, mostly due to conflicting results from clinical trials addressing locoregional LN resections, there is considerable debate if such a sequential metastatic path from the primary tumor over sentinel LN toward peripheral organs, albeit being mechanistically feasible, can outpace hematogenous dissemination and is occurring in a quantitatively meaningful manner to be relevant for disease outcome $(13,14)$.

Therapeutic evaluation of lymphogenous metastasis is handicapped by a lack of suitable preclinical in vivo modeling strategies (15). Although focal and resectable cancer cell line-derived mouse models allow spontaneous metastases to become rate-limiting (16), their nonautochthonous primary tumor growth is associated with a severely perturbed tissue architecture that often involves either collapse or loss 
of intratumoral lymphatics $(17,18)$. Furthermore, they have historically been selected for rapid development of peripheral organ metastases, whose kinetics likely favor a direct hematogenous route of dissemination (19). Conversely, in genetically engineered mouse models (GEMM), de novo tumorigenesis occurs in the natural microenvironment, enabling proper cross-talk of cancer cells with all stromal compartments including the vascular and lymphatic systems (20). As a result, GEMMs have been shown to be superior over cell line-based models in predicting response to vascular-targeted therapies (21). However, most GEMMs, owing to their multifocal nature, have to be sacrificed before developing metastatic disease, hence especially complicating the analysis of sequential metastasis formation.

Angiopoietin-2 (Ang2) is released by endothelial cells (EC) upon angiogenic or inflammatory stimuli and functions as a contextual agonistic or antagonistic ligand of EC-specific Ang1-Tie2 signaling (22). Although Ang2 was originally identified as a key regulator of lymphangiogenesis and lymphatic vessel integrity during embryonic development $(23,24)$, it has in recent years been intensely pursued as a second-generation antiangiogenic candidate molecule (25). Increased Ang2 expression has been associated with poor outcome in a variety of cancers, including melanoma (26). Several preclinical studies, mostly employing reductionist cell line-based tumor models, have demonstrated that Ang2 inhibition reduces primary tumor growth and metastasis by inhibiting tumor angiogenesis, normalizing and stabilizing the vasculature, and altering the inflammatory tumor microenvironment (27-30). By contrast, recent clinical trials assessing Ang2 neutralization in patients with advanced ovarian carcinoma and colorectal cancer failed to demonstrate therapeutic efficacy, which is an intriguing discrepancy that requires further investigation (31-34).

Here, we established a GEMM-derived allograft model that not only displayed functional intratumoral lymphatics, but in which the development of fatal peripheral metastases was driven by a sequential lymphogenous route of tumor cell dissemination. Performing long-term survival studies in neoadjuvant and adjuvant regimens, we revealed differential response patterns of targeting primary tumor- versus metastasis-derived Ang2 and could identify Ang2 as a critical facilitator of tumor cell spread into draining LN and subsequent distant metastatic growth. Mechanistically, LEC transcriptomics up to single-cell resolution and genetic validation unraveled an addiction of intratumoral lymphatics to agonistic Ang2-Tie2 signaling. Accordingly, we found acute Ang2 neutralization to be sufficient to cause lymphatic regression and to prolong overall survival. Collectively, our data unveil a previously unappreciated therapeutic window of Ang2 targeting to inhibit lymphogenous metastasis, which might merit clinical exploitation.

\section{RESULTS \\ Fragment Implantation-Based Mouse Model of Metastatic Melanoma Enables Long-Term Survival Studies and Sustains Intratumoral Lymphatics}

Given limitations of established cell line-based tumor modeling strategies, we set up a novel mouse model of metastatic melanoma (MM), built on MT-ret transgenic mice in which the human RET receptor tyrosine kinase is overexpressed in melanocytes under the control of the mouse metallothionein-I (MT) promoter enhancer (35). These mice spontaneously develop malignant cutaneous melanoma, metastasizing to LN, lung, spleen, kidney, liver, and brain. However, due to fast multifocal tumorigenesis, their clinical endpoint is generally determined by primary tumor load instead of metastatic disease. Converting this multifocal model into a focal model for MM that allows subsequent surgical removal of the primary tumor, we orthotopically implanted fragments of spontaneous MT-ret tumor nodules (diameter of 2-3 $\mathrm{mm}$ ) into syngeneic C57BL/6N wild-type mice (Fig. 1A). Although the initial rate of engraftment was low, successfully engrafted fragments could be biobanked and serially retransplanted for tissue expansion with nearly $100 \%$ efficacy. Following primary tumor resection, animals exhibited a median postsurgical survival of 58 days and developed multiorgan metastases, most frequently in LN, lung, liver, and spleen, recapitulating the diverse metastatic pattern of human disease (Fig. 1B and C). LN metastases were predominantly detected in inguinal and axillary LN (Supplementary Fig. S1A and S1B). Notably, though, when instead transplanting a cell line that was derived from the same initial spontaneous MT-ret tumor, no macroscopic metastases were detectable within 150 days after primary tumor resection (Fig. 1B). Comparative histologic analysis of fragment- and cell line-based tumors to their originating GEMM revealed vastly increased tissue necrosis in the cell line approach (Fig. 1D and E). Assessing non-necrotic tumor regions, we observed only modest differences in blood vascular parameters, such as relative $\mathrm{CD} 31^{+}$vessel area as well as abundance of Desmin ${ }^{+}$and $\mathrm{aSMA}^{+}$pericyte-covered vessels (Fig. 1F; Supplementary Fig. S1C and S1D). In contrast, cell line-based tumors almost completely lacked Lyve $1^{+}$and Vegfr $3^{+}$lymphatics, whereas engrafted fragments were able to maintain the dense intratumoral lymphatic network that is present in spontaneous MT-ret tumor nodules (Fig. 1D and G;

Figure 1. Novel mouse model of MM preserves intratumoral lymphatics. A, Schematic overview depicting the development of the MT-ret-derived fragment transplantation model of MM. B, Kaplan-Meier survival analysis after primary tumor resection of fragment- versus cell line-transplanted MT-ret tumors ( $n=10$ mice). C. Stereomicroscopy images of the four most common metastatic sites at clinical endpoint in the fragment-derived MT-ret model. Fractions of mice with respective organ metastases are indicated. D, Representative hematoxylin and eosin (H\&E) and CD31/Lyve1-stained tumor sections comparing an autochthonous MT-ret tumor (GEMM), an intradermally injected MT-ret cell line, and a transplanted MT-ret tumor fragment. Dashed lines indicate necrotic areas. E-G, Quantification of necrotic area (E), CD31+ vessel area (F), and Lyve1+lymphatic area (G) per tumor. H, qPCR expression analysis of angiogenic markers in MT-ret tumors ( $n=3$ mice). Scale bars, $5 \mathrm{~mm}$ (C) and $200 \mu \mathrm{m}$ (D). Data are shown as mean \pm SD, normalized to the GEMM (F-G) or skin $(\mathbf{H})$ average. Statistical analyses were performed using one-way ANOVA $(\mathbf{E}-\mathbf{G})$ and Student $t(\mathbf{H})$ tests: *, $P<0.05$; ${ }^{* *}, P<0.01$; 悉, $P<0.001 ;$ 
A

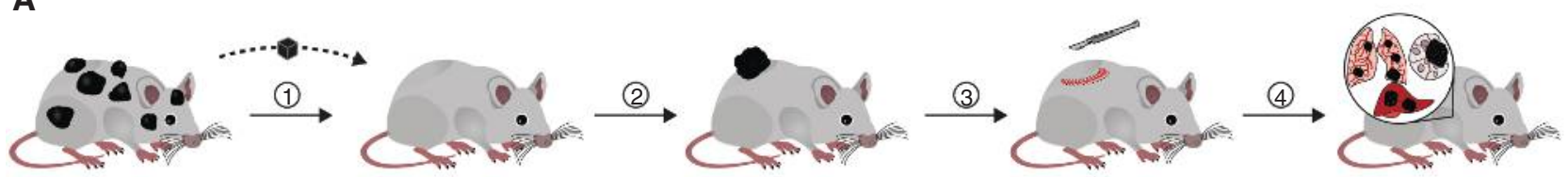

(1) MT-ret fragment transplantation

(2) Primary tumor growth

(3) Primary tumor resection

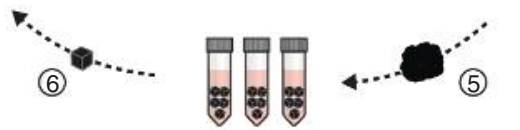

(4) Metastasis formation

(5) Biobanking of tumor fragments

(6) Retransplantation and expansion

B

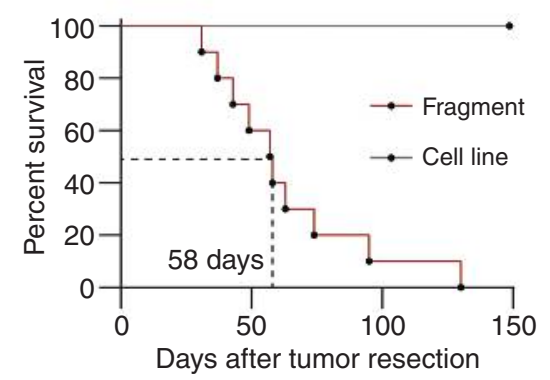

D

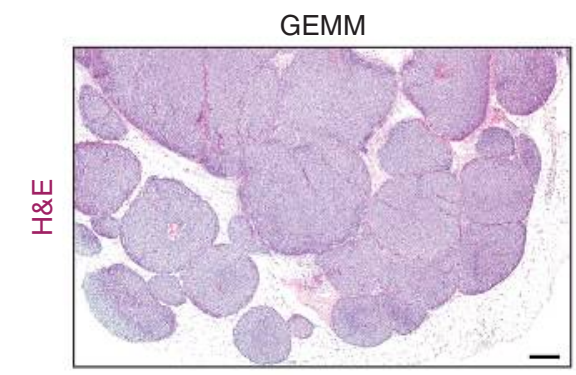

C

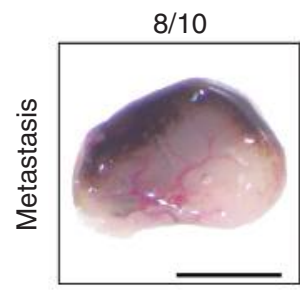

Lymph node

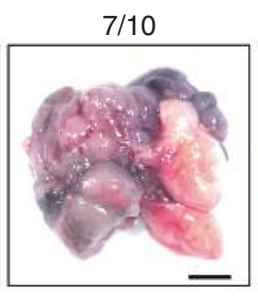

Lung

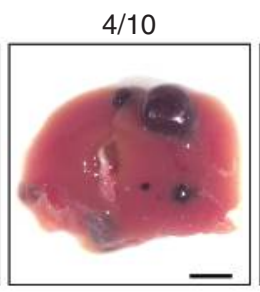

Liver

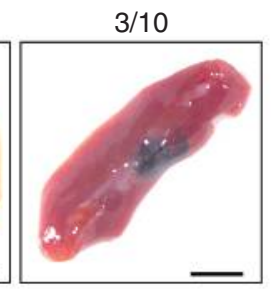

Spleen
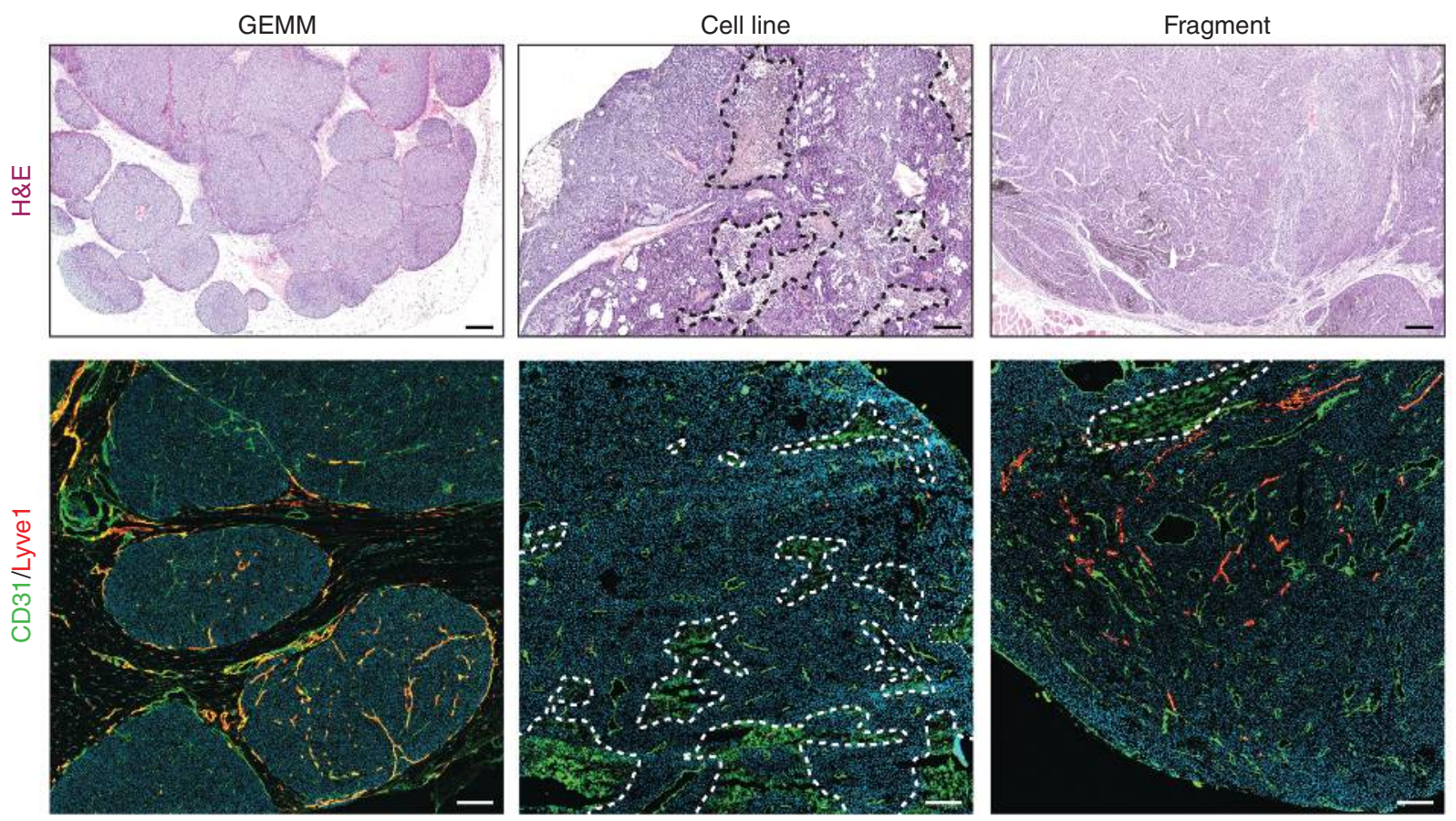

E

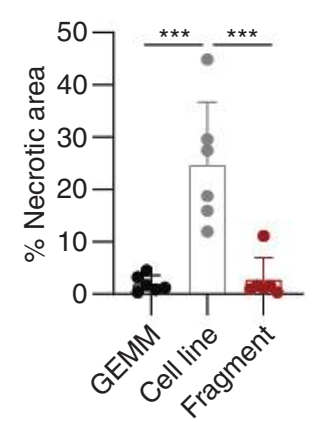

F

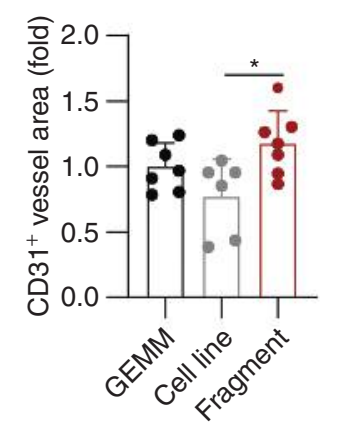

G

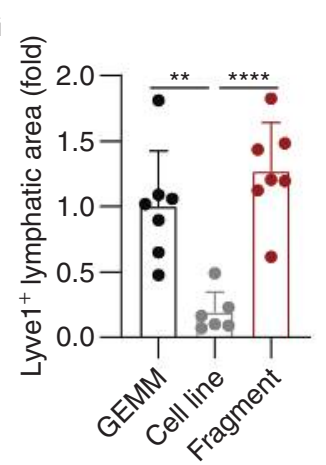

H

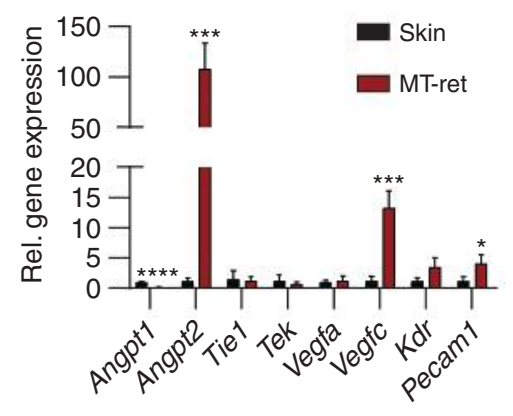


Supplementary Fig. S1E and S1F). Moreover, GEMM-, cell line-, and fragment-derived tumors displayed differential expression of typical vascular pathway genes (Supplementary Fig. S1G). Taken together, avoiding cell culture by direct MTret fragment transplantation allowed us to establish a versatile melanoma model that preserves important histologic and functional characteristics of its originating GEMM. Moreover, large-scale biobanking has made this a standard operating procedure-governed mouse tumor model that may aid preclinical oncology community standardization efforts (15).

\section{Ang2 Is Highly Upregulated in Lymphatic-Rich Melanoma}

Although tumor lymphangiogenesis is an established prognostic indicator for human cutaneous melanoma (36), absence and functional irrelevance of intratumoral lymphatics have been reported for various, mostly cell linebased, murine models (17). Examining quadruple transgenic Tyr-Cre ${ }^{\mathrm{ERT} 2} \times \mathrm{Braf}^{\mathrm{V} 600 \mathrm{E}} \times \mathrm{Pten}^{\mathrm{f} / \mathrm{fl}} \times \mathrm{Ctnnb}^{\mathrm{Exon} 3-\mathrm{f} /+}(\mathrm{TBPC})$ mice that develop malignant melanoma upon topical application of 4-hydroxytamoxifen (4-OHT; Supplementary Fig. S2A and $\mathrm{S} 2 \mathrm{~B}$ ), we could verify the presence of an extensive intratumoral network of Lyve $1^{+}$lymphatics in a second, fully autochthonous melanoma model (Supplementary Fig. S2C). We next investigated key EC growth factor pathways in these models such as VEGF-VEGFR, Ang-Tie, and Notch signaling, by performing microarray expression profiling as well as qPCR analyses of total tissue RNA from tumor samples compared with corresponding healthy skin. In line with our histologic observations, we detected increased expression of Vegfc, a well-described driver of tumor lymphangiogenesis (Fig. 1H; Supplementary Fig. S2D and S2E). Cell sorting of MT-ret tumors revealed that $V e g f c$ was predominantly expressed by $\mathrm{CD}^{-} 5^{-} \mathrm{CD} 31^{+}$EC (Supplementary Fig. S3A and S3B). This was corroborated by reanalysis of two published single-cell data sets $(37,38)$, which also indicated the endothelial compartment as a major source of Vegfc expression in human tumors (Supplementary Fig. S3C and S3D). Intriguingly, Ang2 was the only growth factor that we found even more dominantly upregulated in both MT-ret and TBPC melanoma, rendering it a candidate molecule for further analysis (Fig. 1H; Supplementary Fig. S2D and S2E).

\section{Primary Tumor- but Not Metastasis-Derived Ang2 Is Predictive for Melanoma Survival}

We had previously described circulating Ang2 as a predictive biomarker for metastatic progression and poor survival in $\mathrm{MM}$ that is more sensitive than classic prognostic indicators such as S100 $\beta$ (26), leading us to hypothesize that Ang2 might be functionally linked to melanoma metastasis. Zooming in on its potential site of action, we performed patient survival analyses using Ang2-stained melanoma tissue microarrays from which we stratified all tissue biopsies according to their anatomic origin from a primary versus a secondary site (i.e., we separately analyzed primary tumor- and metastasis-derived samples; Fig. 2A). Remarkably, whereas high primary tumor Ang2 expression was indeed predictive for poor survival, this association could not be found for metastatic expression (Fig. 2B). A similar dichotomy was observed when reanalyzing RNA-sequencing
(RNA-seq) expression data from The Cancer Genome Atlas (TCGA) skin cutaneous melanoma (SKCM) data set (Fig. 2C; Supplementary Fig. S4), altogether indicating that Ang2 plays a dominant role in primary rather than metastatic disease and thus potentially participates in the process of primary tumor cell dissemination.

\section{Neoadjuvant Ang2 Targeting Prolongs Survival}

To investigate if such discrepancy between primary tumorand metastasis-derived Ang2 could be recapitulated in a therapeutic setting, we employed a fully murine Ang2-neutralizing antibody (anti-Ang2) that was first validated in vivo for its antiangiogenic and vessel-normalizing effects in the postnatal retinal angiogenesis assay (Supplementary Fig. S5A-S5D). Moreover, we detected elevated concentrations of circulating Ang2 following anti-Ang2 treatment, indicating increased serum persistence of antibody-bound compared with free Ang2 as previously shown for another Ang2-binding antibody (ref. 39; Supplementary Fig. S5E). Consistent with the correlative patient data, anti-Ang2 therapy failed to improve overall survival when administered in a postsurgical adjuvant regimen, i.e., targeting metastasis-derived Ang2 (Fig. 2D and E; Supplementary Fig. S6A). By contrast, presurgical neoadjuvant intervention led to a dramatic survival benefit with more than $50 \%$ of treated animals being long-term survivors (Fig. 2F; Supplementary Fig. S6A). Excluding off-target effects, we could validate this finding by implanting MT-ret fragments into mice with global and constitutive Ang2 deletion (Ang2 ${ }^{\mathrm{KO}}$ ), further indicating that such antibody treatment acted by blocking host-derived stromal Ang2 (Fig. 2G). In line with previous publications, neoadjuvant therapy or genetic deletion of Ang2 resulted in a modest primary tumor growth delay (refs. 27, 40; Supplementary Fig. S6B-S6D). Nevertheless, by strictly resecting size-matched primary tumors, we compensated for the differences in growth kinetics in our experiments, thereby making the observed survival advantage robust and fully independent of variations in primary tumor size. PET/CT imaging and gross necropsy analysis at clinical endpoint revealed no difference in the distribution of metastatic organ sites upon adjuvant treatment (Supplementary Fig. S6E), whereas LN lesions were to our surprise completely absent in both the neoadjuvant and the genetic setting (Supplementary Fig. S6F-S6H), hinting at a critical function of primary tumor-derived Ang2, particularly for lymphatic dissemination.

\section{Therapeutic Responses of Anti-Ang2 Are Independent of Intravasation and Immune Function}

We next conducted a series of experiments aimed at mechanistically deciphering the survival benefit of targeting primary tumor-derived Ang2. Blood flow cytometry, plating experiments, and quantification of melanoma cell-specific transcripts detected no differences in circulating tumor cell numbers, indicating that Ang2 blockade was not inhibiting melanoma cell intravasation (Supplementary Fig. S7A-S7H). By contrast, experimental tail-vein metastasis assays with antiAng2-pretreated animals revealed decreased lung metastasis, thereby verifying a previously reported reduction in tumor cell extravasation (ref. 27; Supplementary Fig. S8A-S8G). 
A
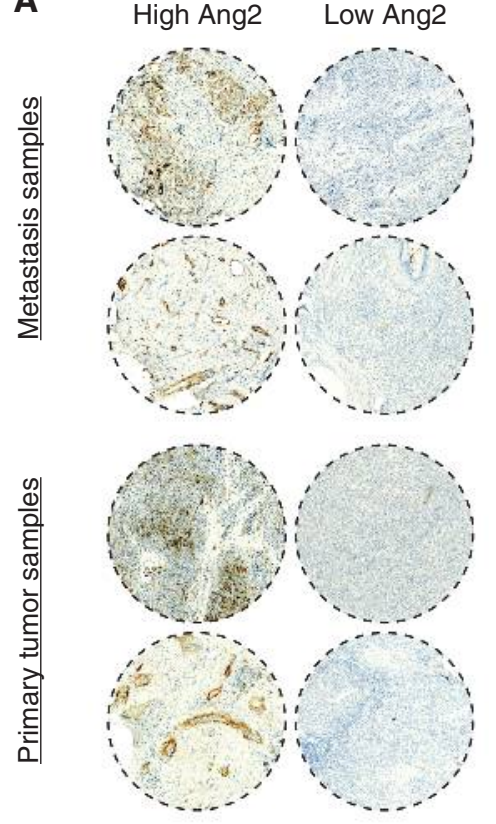

B
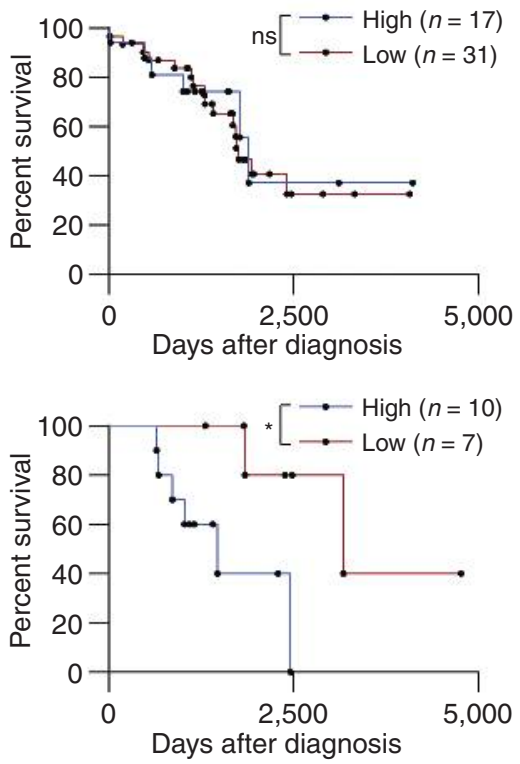

C
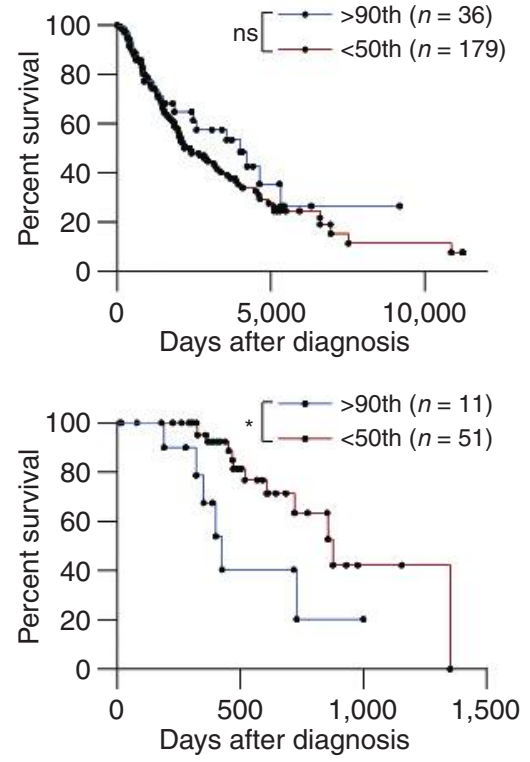

D

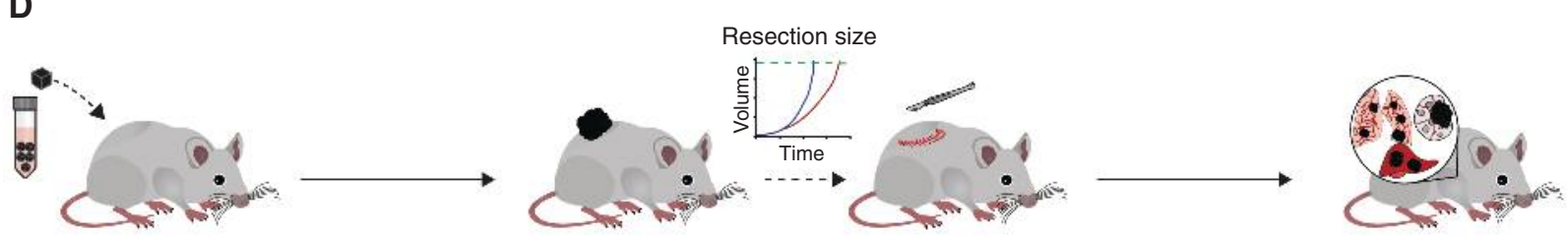

Adjuvant

IgG vs. anti-Ang2

Neoadjuvant IgG vs. anti-Ang2

Genetic Ang2 ${ }^{\text {WT }}$ vs. Ang2 $2^{\mathrm{KO}}$

E

Adjuvant

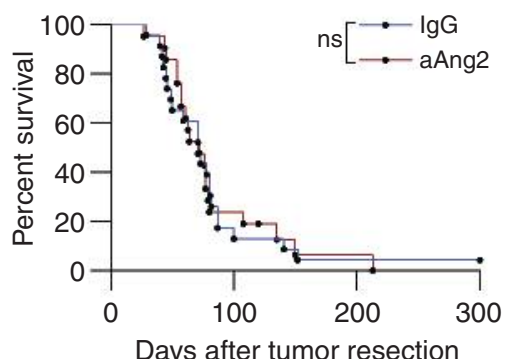

$\mathbf{F}$

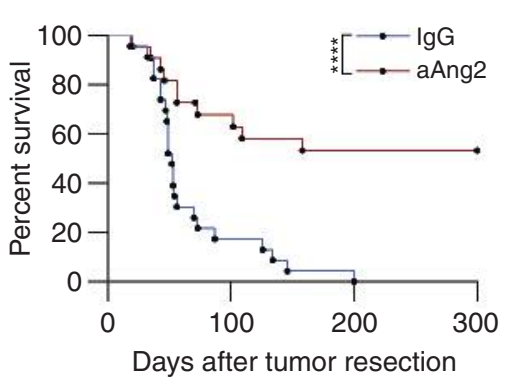

G

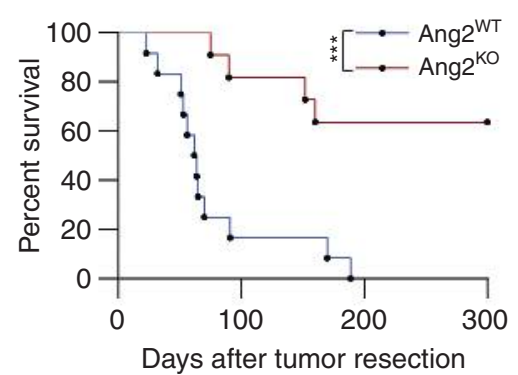

Figure 2. Targeting primary tumor versus metastasis-derived Ang2. A, Representative Ang2-stained human tissue microarray sections of primary tumor- and metastasis-derived melanoma specimens. B-C, Kaplan-Meier survival analyses of patients with melanoma with high and low Ang2 expression according to IHC [B; expression score 0-1 (low) vs. 2-3 (high)] or mRNA expression [C; TCGA-SKCM data set, > 90th (high) vs. < 50th (low) expression percentile]. Subgroup-specific n numbers are indicated in brackets. Top plot, metastasis samples. Bottom plot, primary tumor samples. D, Schematic overview depicting antibody-mediated and genetic targeting strategies of Ang2 in the MT-ret model. Detailed treatment regimens are depicted in Supplementary Fig. S6A. E-G, Kaplan-Meier survival analysis of MT-ret fragment-transplanted mice treated with anti-Ang2 or control-IgG in an adjuvant (E) or neoadjuvant (F) regimen and of MT-ret-fragment transplanted Ang $2^{\mathrm{KO}}$ and Ang $2^{\text {WT }}$ mice $(\mathbf{G})$. Statistical analyses were performed using Log-rank test: ns, not significant; *, $P<0.05 ;$ 
Yet, changes in extravasation could mechanistically not explain the observed absence of LN metastases upon neoadjuvant Ang2 blockade, because cancer cells are thought to enter LNs via the lymphatic route (2). In addition, FACSand histology-based tumor immune phenotyping confirmed a recently described increase of intratumoral $\mathrm{CD}^{+} \mathrm{T}$-cell infiltrates upon Ang2 inhibition, especially in combination with VEGFR2 blockade (ref. 41; Supplementary Figs. S9A and S10A-S10D). However, neoadjuvant anti-Ang2 treatment in severely immunodeficient NOD/SCID gamma (NSG) mice implanted with MT-ret fragments displayed a similar survival benefit as observed in immunocompetent $\mathrm{C} 57 \mathrm{BL} / 6 \mathrm{~N}$ animals, thereby excluding a primarily immune-mediated mechanism of action (Supplementary Fig. S10E-S10G).

\section{Lymphendothelial CCL21 Is Depleted upon Ang2 Inhibition}

In order to gain better molecular insight, we carried out total tumor RNA microarray analysis to identify differentially regulated genes and pathways following anti-Ang2 treatment, which revealed Ccl21 family members among the top five downregulated transcripts (Fig. 3A; Supplementary Fig. S11A-S11D; Supplementary Table S1). In addition, CCL21 was discovered as a prominently decreased chemokine by performing inflammatory cytokine profiling with whole tumor lysates upon either antibody-mediated or genetic Ang2 neutralization (Fig. 3B and C; Supplementary Fig. S12A and S12B), a finding that we subsequently confirmed by ELISA (Fig. 3D). By contrast, Vegfc and Vegfd expression remained unchanged upon anti-Ang2 treatment, suggesting that therapeutic responses to Ang2 blockade were likely independent of the VEGFC/VEGFD-VEGFR3 signaling axis (Supplementary Fig. S13A and S13B). Within most nonlymphoid organs, initial lymphatics are thought to be the primary source of CCL21, which binds to its cognate receptor CCR7 on several immune-cell types, thereby recruiting them toward the lymphatic vasculature and finally toward the draining $\mathrm{LN}$ to initiate an immune response (42). Investigating its source in neoplastic tissue, we FACS-sorted MT-ret tumors into multiple cellular components and could in fact pinpoint Ccl21 expression exclusively to LEC (Fig. 3E; Supplementary Fig. S13C), which indicated that reduced CCL21 levels after Ang2 neutralization might have resulted from either LEC-specific Ccl21 downregulation or a decrease in LEC numbers. More recently, CCL 21 was described to stimulate $\mathrm{LN}$ metastasis via chemoattraction of CCR $7^{+}$tumor cells in multiple cancer types $(43,44)$. Indeed, we found CCR7 expression and surface presentation by MT-ret melanoma cells (Fig. 3E and F), indicating presence of an active lymphatic metastasis-promoting CCL21-CCR7 axis in this model.

\section{Ang2 Neutralization Disrupts Intratumoral Lymphatics}

In view of similar expression levels of major Ang-Tie pathway components in tumor LEC and blood vascular EC (BEC) such as Angpt2, Tek, and Tie1 (Fig. 3E; Supplementary Fig. S13D), we asked if anti-Ang2 treatment would trigger differential responses in the lymphatic versus blood vascular system. Strikingly, when analyzing size-matched primary tumors, we observed only minor effects on tumor blood vessels, which, though in line with previously reported vascular maturation phenotypes (40), were significant only upon antibody-mediated and not genetic Ang2 neutralization (Fig. 3G-J; Supplementary Fig. S14A and S14B). By contrast, Lyve $1^{+}$intratumoral lymphatics were severely decreased upon anti-Ang2 therapy as well as in Ang $2^{\mathrm{KO}}$ mice (Fig. 3G and $\mathrm{K}$ ), which explicated the observed reduction in intratumoral CCL21 levels and suggested a more critical Ang2 dependency of tumor LEC compared with BEC. Confirming that we detected not just Lyve1 marker downregulation, but structural and thereby functional disruption of tumor lymphatics, we analyzed drainage of intratumorally injected low- and high-molecular-weight dyes toward inguinal and axillary LN (Fig. 3L and M; Supplementary Fig. S14C-S14E). The data unveiled almost-complete obstruction of tumor drainage upon antibody-mediated or genetic Ang2 targeting, providing a mechanistic explanation for the noticed inhibition of $\mathrm{LN}$ metastasis.

\section{Anti-Ang2 Therapy Efficiently Blocks Lymphatic Metastasis in Multiple Tumor Models}

To confirm these findings in a fully autochthonous melanoma model, we treated 4-OHT-induced TBPC mice with anti-Ang2 during primary tumor growth and examined all tumors as soon as they surpassed a cutoff volume of 400 $\mathrm{mm}^{3}$ (Supplementary Fig. S15A and S15B). Similar to the MT-ret model, we found the tumor blood vasculature to be largely unaffected in size-matched melanomas, whereas intratumoral lymphatics were greatly diminished upon Ang2 blockade (Supplementary Fig. S15C and S15D). Likewise, gross necropsy at time of primary tumor analysis revealed a considerable reduction of pigmented LN lesions, substantiating a functional link between Ang2 and lymphatic dissemination (Supplementary Fig. S15E). Indicating a similar

Figure 3. Ang2 neutralization disrupts intratumoral lymphatics. A, Microarray heatmap depicting the top 10 differentially expressed genes in MT-ret tumors after neoadjuvant Ang2 blockade. A heat map indicating all differentially expressed genes is shown in Supplementary Fig. S11C. B and C, Proteome profiler antibody-based array assessing inflammatory cytokines upon neoadjuvant or genetic Ang2 targeting. Pooled MT-ret tumor lysates of 4 to 6 mice were probed on each membrane. B, Cropped membranes indicating Ang2 (dashed orange line) and CCL21 (dashed violet line) dots. Uncropped membranes are shown in Supplementary Fig. S12A and S12B. C, Densitometric quantification of Ang2 and CCL21. D, ELISA quantification of CCL21 in individual MT-ret tumor lysates. E, qPCR expression analysis of FACS-sorted MT-ret tumor cell populations (TC, tumor cell enriched; $n=3$ mice). F, Flow cytometry analysis of MT-ret tumor cells for CCR7 surface expression. G, Representative CD31/Lyve1 stained MT-ret tumor sections. H-K, Relative quantification of $\mathrm{CD} 1^{+}$vessel area $(\mathbf{H})$, Desmin (I), and aSMA (J) covered vessels and Lyve $1^{+}$lymphatic area (K). L, On the left, schematic overview of the Evans Blue tumor drainage assay. On the right, stereomicroscopy images of tumor-draining axillary and inguinal LN upon Ang2 inhibition. M, Relative quantification of tumor-drained Evans Blue. Scale bars, $200 \mu \mathrm{m}$. Data are shown as mean \pm SD, normalized to the IgG and Ang2WT average (C, $\mathbf{H}-\mathbf{K}$, and $\mathbf{M})$ or Actb expression (E). Statistical analyses were performed using Student's $t(\mathbf{A}$ and $\mathbf{D})$, one-way ANOVA (E), and Mann-Whitney U (H-K and $\mathbf{M})$ tests. ns, not significant;

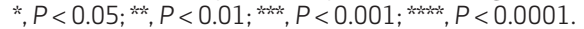




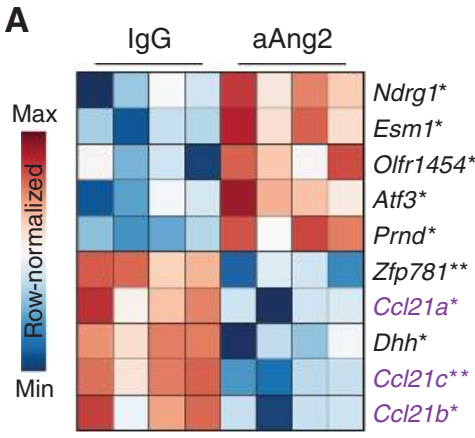

D

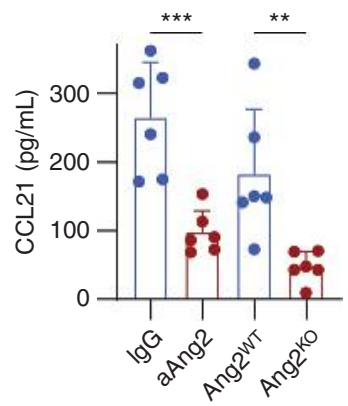

G
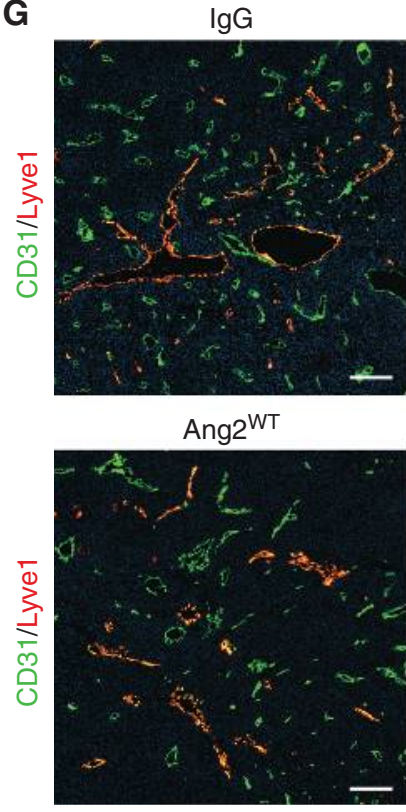

L

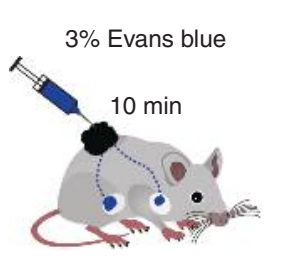

E
B

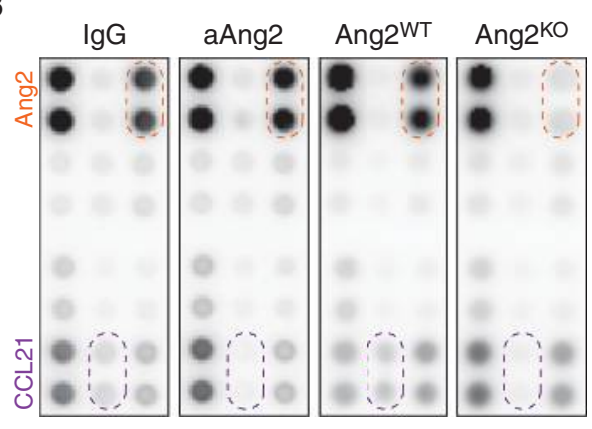

C $\begin{array}{ll}=\text { IgG } & \text { Ang2WT } \\ \text { aAng2 } & \text { Ang2KO }\end{array}$

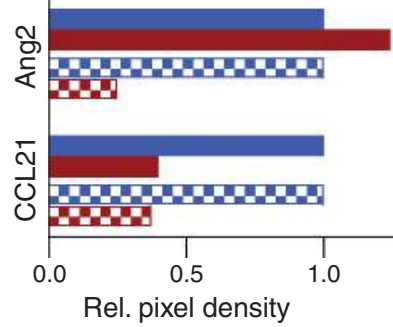

F $\quad-\operatorname{CCR} 7$

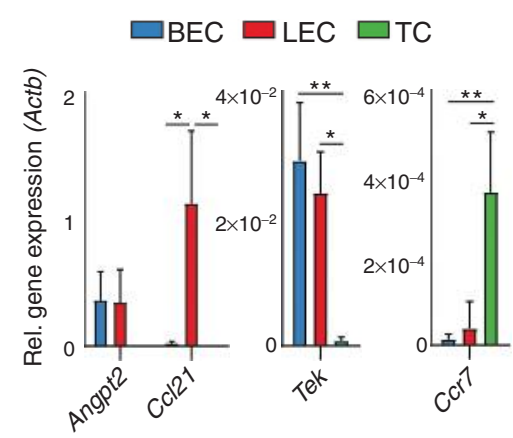

— Isotype ctrl

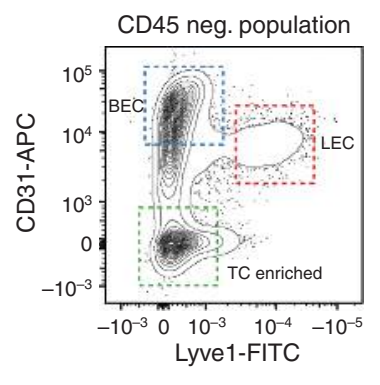

H

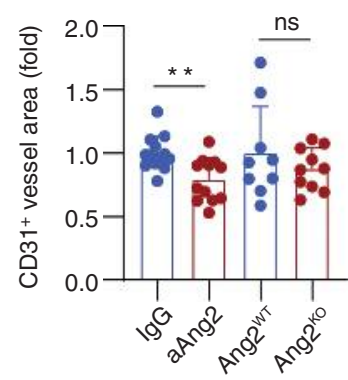

K

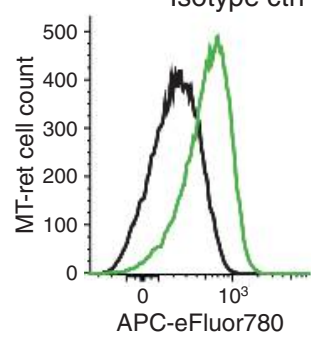

I

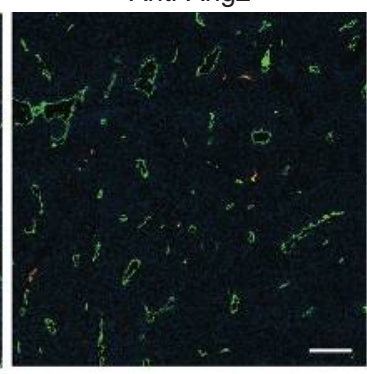

$\mathrm{Ang}^{\mathrm{KO}}$
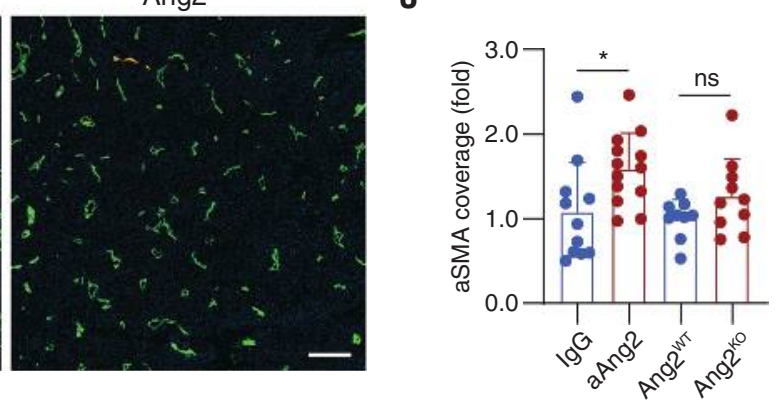

M
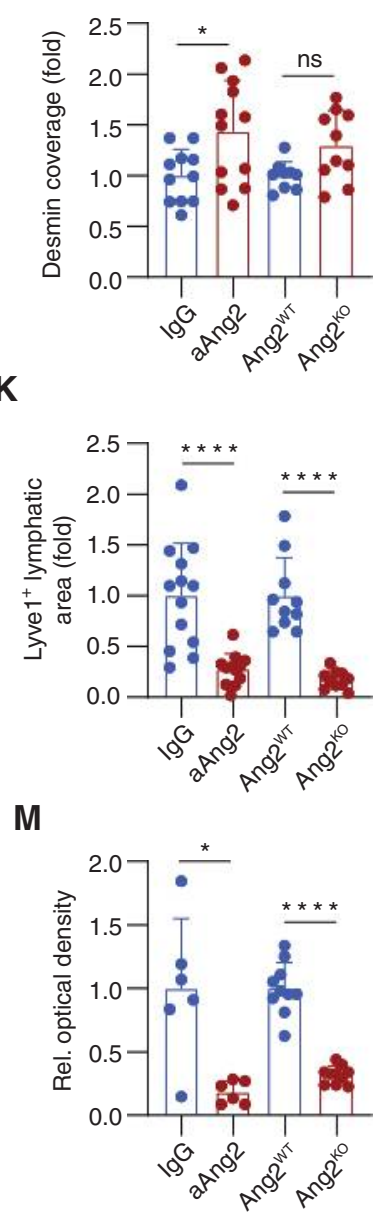
A

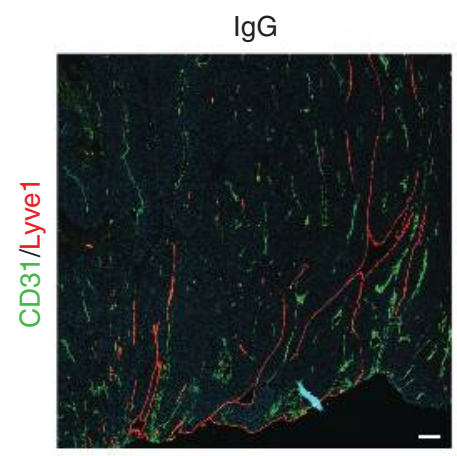

B

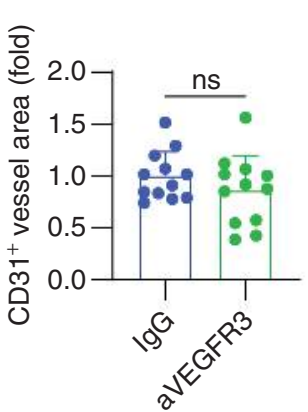

C
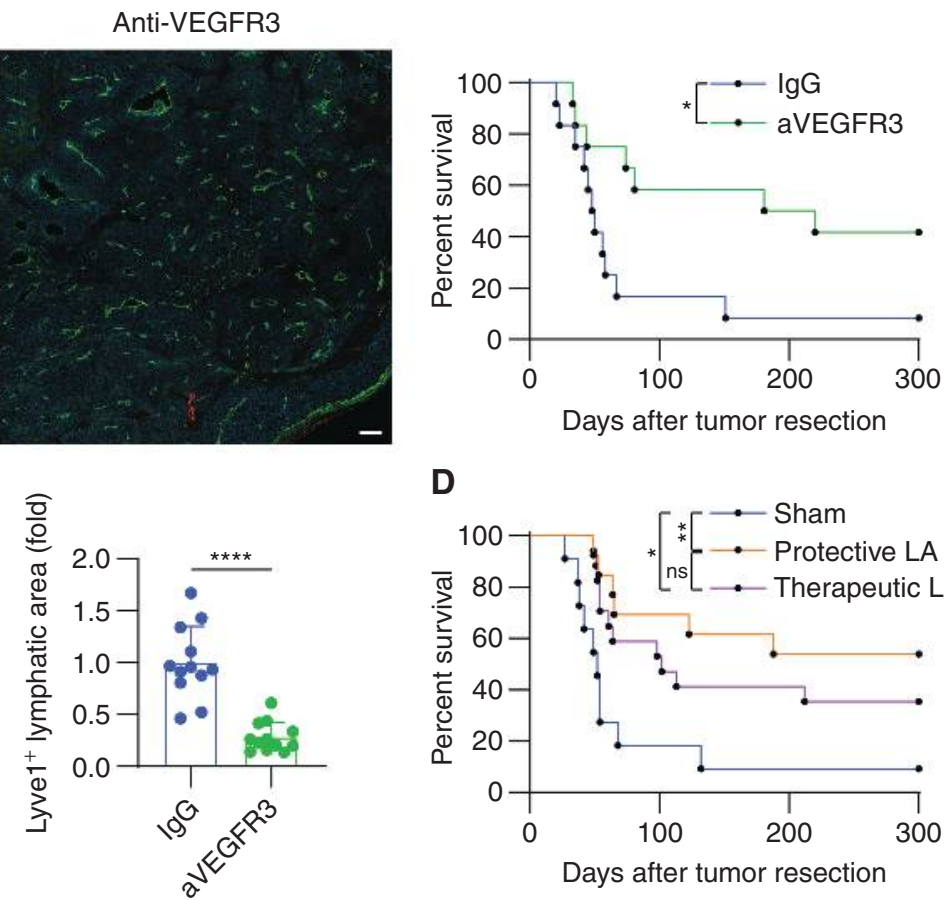

D

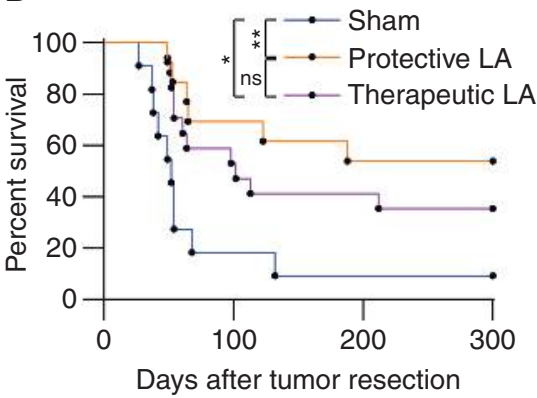

Figure 4. Lymphogenous route of metastasis determines long-term survival. A-D, Experimental inhibition of lymphatic metastasis in the MT-ret model via antibody-mediated VEGFR3 blockade during primary tumor growth or LA of all tumor-draining LN. Detailed treatment regimens are depicted in Supplementary Fig. S20A. A, Representative CD31/Lyve1 stained MT-ret tumor sections. B, Relative quantification of CD31+ vessel and Lyve ${ }^{+}$ lymphatic area. Data are shown as mean \pm SD, normalized to the IgG average. C, Kaplan-Meier survival analysis upon VEGFR3 blockade. D, Kaplan-Meier survival analysis upon protective or therapeutic LA. Scale bars, $200 \mu \mathrm{m}$. Statistical analyses were performed using Mann-Whitney U (B) and Log-rank

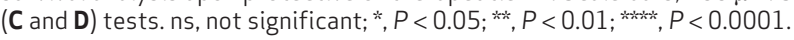

relationship in human disease, we detected a significant correlation between Ang2 expression and the amount of tumor-associated lymphatics by scoring Ang2- and Podoplanin (D2-40)-stained tumor sections of patients with cutaneous melanoma (Supplementary Fig. S16A and S16B).

Because we also found a similar correlation in patients with breast cancer (Supplementary Fig. S16C and S16D), we next studied the efficacy of neoadjuvant Ang2 targeting in an orthotopic mammary carcinoma model (Supplementary Fig. S17A and S17B). Confirming vascular results obtained in melanoma models, tumor blood vessels were only weakly affected, whereas intratumoral lymphatics were significantly reduced in anti-Ang2-treated 4T1 tumors (Supplementary Fig. S17C and S17D). Furthermore, we traced organ metastases 2 weeks after primary tumor removal via bioluminescence imaging, which uncovered not only decreased LN but also reduced distant metastasis formation (Supplementary Fig. S17E-S17F).

Lastly, assessing a third tumor entity, we performed presurgical Ang2 neutralization and survival analysis in the Lewis lung carcinoma (LLC) model (Supplementary Fig. S18A and S18B). Although LLC tumors contained only a few intratumoral lymphatics at baseline, we could still detect a significant reduction upon Ang2 blockade (Supplementary Fig. S18C and S18D). In addition, anti-Ang2-treated animals displayed reduced LN metastasis at clinical endpoint. Surprisingly though, neither distant metastasis formation (primarily lung lesions) nor overall survival were affected, contrary to the therapeutic results that we had gained in melanoma and breast cancer models (Supplementary Fig. S18E and S18F). This hinted at either an inefficacy of the anti-Ang2 treatment or other mechanisms of metastasis that deserve further investigation in subsequent experiments.

\section{Lymphogenous Route of Metastasis Can Be the Major Determinant of Long-Term Survival}

Integrating the above-observed model-dependent differential response patterns of Ang2 inhibition in terms of distant metastasis formation and long-term survival, we found that therapeutic efficacy correlated with intratumoral levels of Ang2, CCL21, and VEGFC (Supplementary Fig. S19A-S19C). We hypothesized that neoadjuvant anti-Ang2 did not primarily affect hematogenous dissemination and might instead be beneficial in tumors that metastasize via a lymphogenous route, i.e., in which survival-limiting peripheral metastasis is dependent on earlier LN colonization, a metastatic path that was only recently proven to exist in mice (12). Confirming this hypothesis, we selectively blocked only lymphatic dissemination in the MT-ret model using first a chemical approach by inhibiting tumor lymphangiogenesis with a VEGFR3blocking antibody (anti-VEGFR3) during primary melanoma growth (Fig. 4A and B; Supplementary Fig. S20A-S20C). Secondly, we performed surgical blockade of LN metastasis via subtotal lymphadenectomy (LA) of all tumor-draining 
$\mathrm{LN}$, either before fragment implantation (protective LA) or following primary tumor resection (therapeutic LA; Supplementary Fig. S20A and S20D). Both chemical and surgical approaches resulted in a substantial survival benefit in the MT-ret model, thereby not only phenocopying presurgical anti-Ang2 therapy, but also elucidating lymphogenous metastasis as the key determinant for long-term survival (Fig. 4C and D). Moreover, surgical removal of tumor-draining LN was sufficient to significantly limit distant metastasis formation (Supplementary Fig. S20E). By contrast, neither protective nor therapeutic LA increased overall survival in the LLC model, clearly illustrating that hematogenous and not lymphogenous spread of LLC cells determined fatal distant metastasis formation (Supplementary Fig. S21A-S21C). Collectively, the data suggest that the efficacy of neoadjuvant Ang2 neutralization in prolonging long-term survival is predominantly dependent on a tumor's relative potential to metastasize via a lymphogenous contrary to a hematogenous route of dissemination.

\section{Intratumoral LECs Are a Heterogeneous Cell Population}

Assessing the transcriptional heterogeneity of tumor LEC and BEC, equal numbers of intratumoral CD $31^{+}$Lyve $1^{-}$and $\mathrm{CD} 31^{+}$Lyve $1^{+}$cells were isolated and pooled from 10 individual MT-ret tumor-bearing mice for conducting singlecell transcriptomics. mRNA libraries were prepared using the $10 \times$ Genomics platform and sequenced on the Illumina HiSeq4000 system. Following quality filtering, we obtained data for 7,635 cells that could be clustered via Seurat into 12 distinct subpopulations (Fig. 5A). Heat-map analysis illustrating the top 10 cluster-discriminative genes as well as t-distributed stochastic neighbor embedding ( $t$-SNE) indicated close transcriptional similarity between multiple subpopulations that could consequently be aggregated into five larger cluster families (Fig. 5B). Based on known marker gene expression, these could be identified as LEC, BEC, and cycling EC (CEC) as well as melanoma and mesenchymal cells (representing two contaminating cell clusters; Fig. 5C; Supplementary Fig. S22A-S22C). Focusing solely on BEC, top differentially expressed genes and gene ontology (GO) analyses revealed considerable transcriptomic diversity between the four identified BEC subclusters (Supplementary Fig. S23A and S23B). We could further annotate these as tip-like and stalk-like populations, corroborating a recently published single-cell-based nomenclature of tumor-derived EC (ref. 45; Supplementary Fig. S23C). Unexpectedly, we also detected five distinct LEC subpopulations based on differential gene expression (Supplementary Fig. S24A). Moreover, GO analysis pointed toward distinct LEC subcluster-specific biological functions such as cellular motility, hypoxia signaling, inflammatory response, antigen presentation, and protein biosynthesis (Supplementary Fig. S24B), highlighting a previously unappreciated heterogeneity within the intratumoral LEC compartment.

\section{Cluster-Discriminative Expression of Ang-Tie Pathway Genes Indicates Tumor LEC-Specific Functions}

Aiming at decoding the diverging cellular sensitivity of LEC and BEC toward Ang2 neutralization, we mapped major com- ponents of the Ang-Tie pathway over all tumor EC subclusters (Fig. 5D). Although Angpt2 expression was cluster-specific (enriched in tip-like BEC and hypoxic LEC), we observed a matching degree of transcriptomic heterogeneity in both EC types. Tie $1^{+}$and $\operatorname{Itg} b 1^{+}$cells were homogenously scattered over all EC, thus similarly not hinting at Tie1 or integrin receptor functions that would be unique for either EC population. Conversely, an EC-type distinctive transcriptomic pattern was discovered for Tek expression. Whereas Tek $k^{+}$cells were confined to stalk-like BEC, confirming published literature (46, 47), they were more evenly dispersed among all LEC subpopulations, implying broader Tie2 dependency of tumor LEC. In addition, Ptprb (encoding VE-PTP), which had recently been identified as the determining factor for antagonistic Ang2 function on Tie2, was strongly expressed by BEC and largely absent in $\operatorname{LEC}(48,49)$. Combined with the fact that intratumoral lymphatics, not being pericyte covered, lack any natural source for the agonistic Tie2-ligand Ang1, this prompted us to postulate that tumor LEC might instead rely on agonistic Ang2-Tie2 signaling.

\section{Tumor Lymphendothelial Tie2 Deletion Phenocopies Ang2 Inhibition}

Cell-fate tracing of MT-ret fragments, which had been implanted into $\mathrm{mTmG}$-reporter mice, revealed that intratumoral blood and lymphatic vessels consisted of host- instead of fragment-derived cells (Supplementary Fig. S25A-S25D). This allowed investigation of lymphatic Tie2 dependency by implanting MT-ret fragments into Prox1-Cre ${ }^{\text {ERT2 }} \times$ Tie $^{\text {f//fl }}$ $\left(\mathrm{Tie} 2^{\mathrm{iLECKO}}\right)$ mice that were treated with tamoxifen during primary tumor growth to induce LEC-specific Tie2 deletion (Supplementary Fig. S26A). Vascular analysis of size-matched tumors revealed significantly diminished lymphatics, but unaffected blood vessels in Tie ${ }^{\text {iLECKO }}$ mice (Fig. 5E and F; Supplementary Fig. S26B-S26F). Tie2 deletion hence phenocopied neoadjuvant Ang2 neutralization. Analogously, we noticed declined intratumoral CCL21 levels, reduced LN metastasis at clinical endpoint, and most importantly increased postsurgical survival (Fig. 5G; Supplementary Fig. S26G and S26H), overall not only demonstrating an essential role of LEC-expressed Tie2, but also suggesting that antiAng2 treatment blocks agonistic Ang2-Tie2 interaction on tumor LEC.

\section{Acute Ang2 Blockade Drives an Apoptotic Gene Signature in Tumor LEC}

Being unable to purify viable LEC after long-term neoadjuvant anti-Ang2 therapy, owing to the near-complete disappearance of intratumoral lymphatics, we switched to an acute treatment regimen to study tumor LEC-specific molecular changes. Hence, we FACS-isolated LEC with high purity from MT-ret tumor-bearing mice, 12 hours after injecting a single dose of anti-Ang2, and subsequently performed bulk RNAseq for transcriptional landscaping (Fig. 6A; Supplementary Fig. S27A). This approach revealed downregulation of Ccl21a expression in tumor LEC upon short-term Ang2 blockade, a finding that was subsequently validated in vitro in cultured human dermal LEC (Supplementary Fig. S27B and S27C). Principal component analysis of RNA-seq data demonstrated treatment group-specific clustering, reflecting global 
A

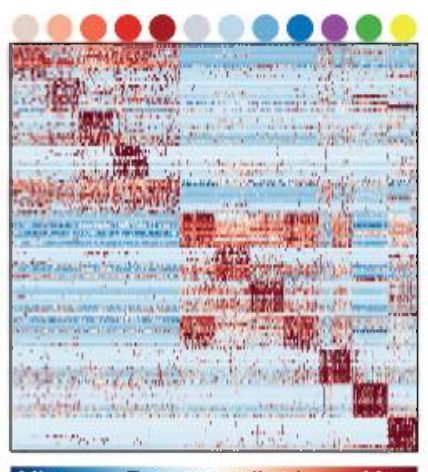

Min Row-normalized Max
Lymphatic EC (LEC)
Blood vascular EC (BEC)
L1 Gja4 ${ }^{\text {hi }}(n=2,746)$
L2 $\mathrm{Nmb}^{\text {hi }}(n=790)$
(L3. $\mathrm{Ccl}^{\mathrm{hi}}(n=95)$
(4) $C d 74^{\mathrm{hi}}(n=95)$
B1 Esmt $1^{\text {hi }}(n=2,340)$
B2 $\operatorname{Lrg} 7^{\text {hi }}(n=378)$
B3 Stmn $2^{\text {hi }} T c f 15^{\text {hi }}(n=136)$
(3) Rpl35 $5^{\mathrm{hi}}$ Malat $^{\mathrm{lo}}(n=46)$
(B) Lgals $1^{\mathrm{hi}}$ Malat1 ${ }^{10}(n=133)$
Cycling EC (CEC)
C. $(n=450)$
Tumor (TC)
(T) $(n=383)$
Mesenchymal (MC)
M $(n=43)$

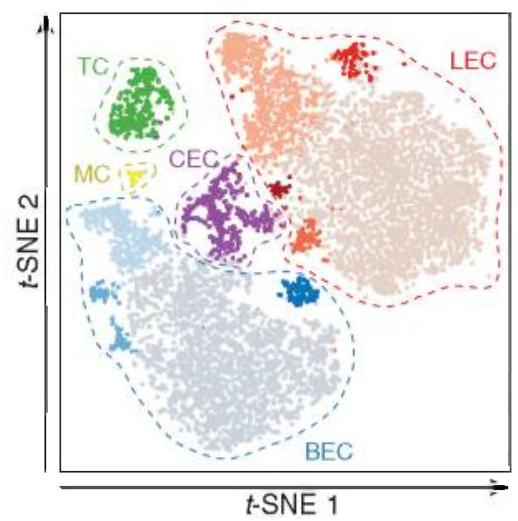

\section{D}

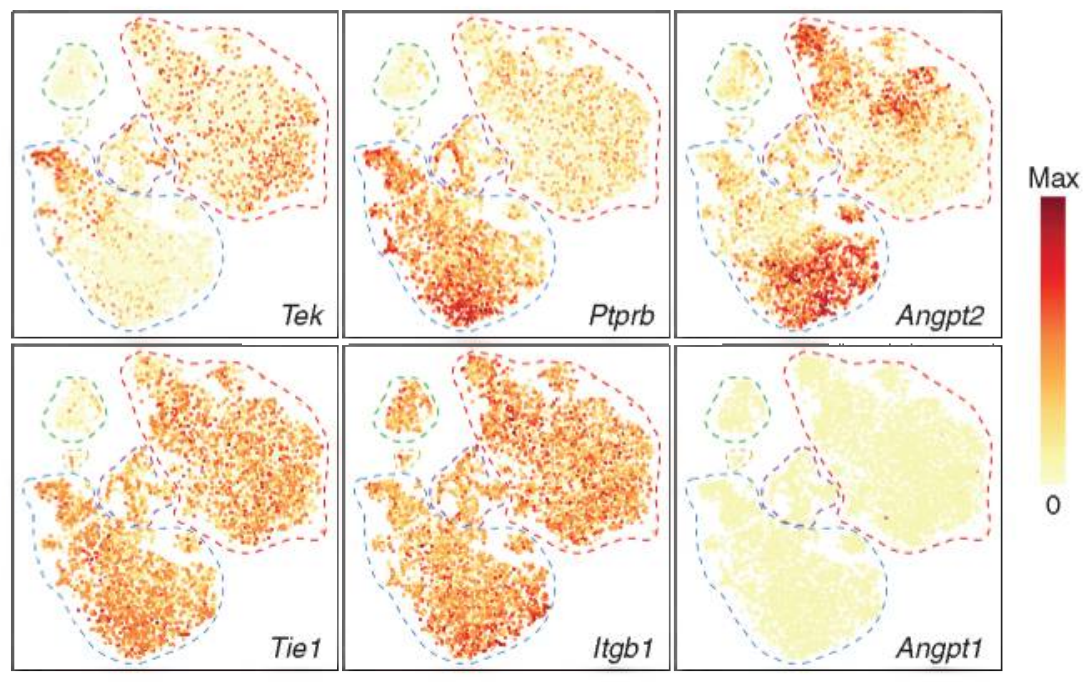

E

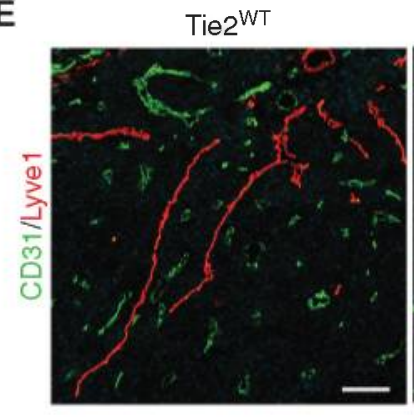

Tie2 $2^{\text {iLECKO }}$

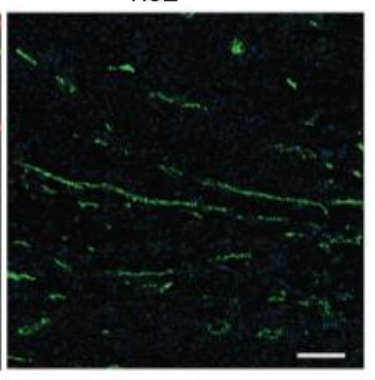

F

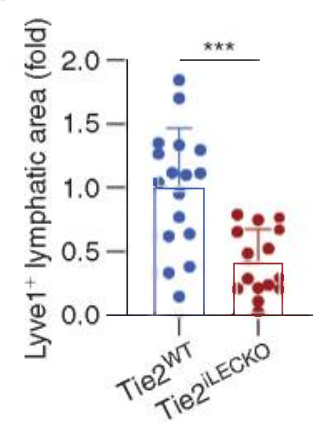

C

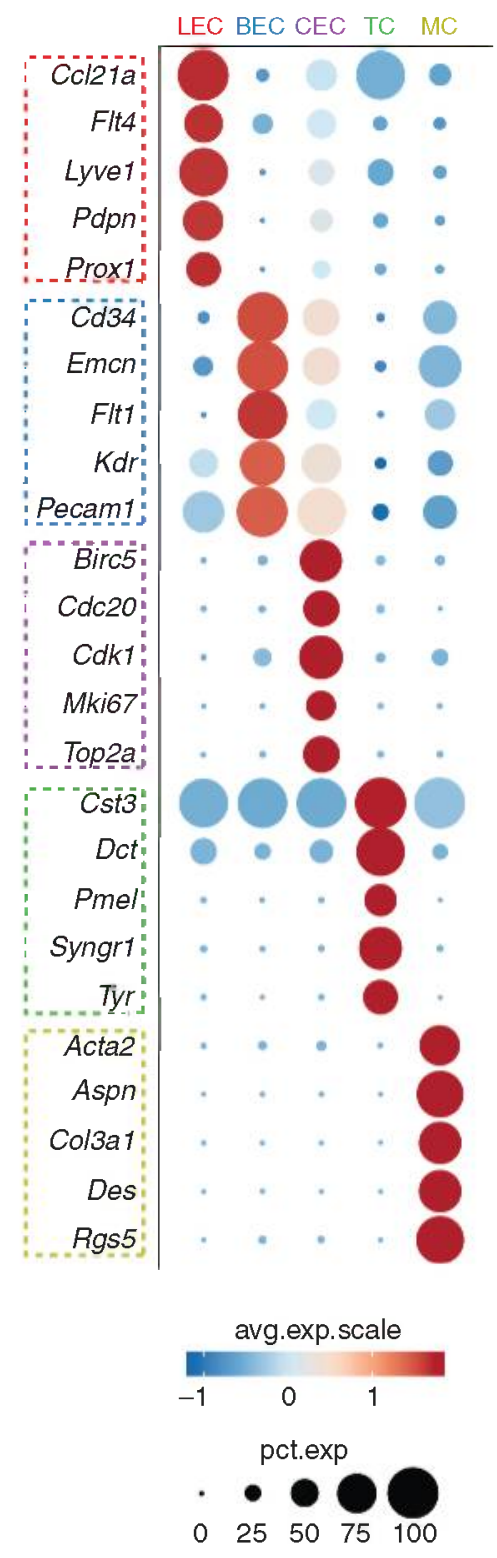

G

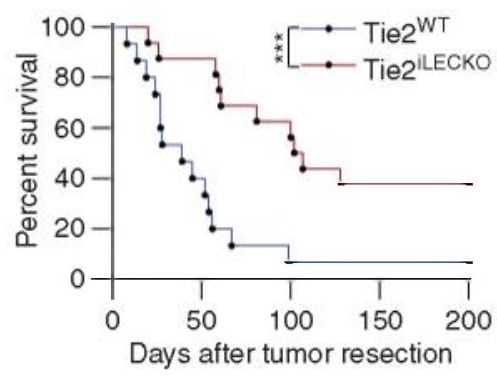


alterations in the LEC transcriptome upon short-term Ang2 inhibition (Fig. 6B). Conducting unbiased gene set enrichment analysis (GSEA), we surprisingly found Apoptosis as the sole hallmark gene set that was significantly enriched in tumor LEC from anti-Ang2-treated animals (Fig. 6C-E). By contrast, control LEC displayed an enrichment of E2F target and Notch signaling genes, which have previously been implicated as regulators of lymphatic growth and function $(50,51)$. Ingenuity pathway analysis (IPA; focusing on differentially regulated genes; $\left.P_{\text {adj }}<0.05\right)$ not only supported the GSEA data but also validated specificity of antibody-mediated Ang2 neutralization by identifying both prominent upregulation of various cell death-related pathways and biofunctions as well as a significant downregulation of canonical Angiopoietin signaling (Fig. 6F; Supplementary Fig. S27D and S27E). Furthermore, immunofluorescence analysis unveiled increased activation of Caspase-3 (Casp3) and PARP1 in tumor LEC of anti-Ang2-treated mice (Fig. 6G and H; Supplementary Fig. S27F and S27G), altogether suggesting active regression of intratumoral lymphatics following acute Ang2 inhibition. By contrast, acute VEGFR3 blockade neither resulted in changed LEC-specific gene expression of apoptotic regulators nor did it lead to increased presence of cleaved Casp $3^{+}$and cleaved $\mathrm{PARP}^{+}$intratumoral lymphatic vessels (Supplementary Fig. S27H-S27K).

\section{Presurgical Anti-Ang2 Pulses Are Sufficient to Prolong Survival}

Peak lymphatic tumor cell dissemination has been associated with surgical stress at time of primary tumor resection, classifying it as a late-stage iatrogenic event (52). Hence, having established pronounced molecular changes after only one dose of anti-Ang2, we sought to test the therapeutic efficacy of presurgical Ang2 neutralization within a very condensed and clinically realistic treatment window (Fig. 6I). Remarkably, although two presurgical pulses of anti-Ang2 were sufficient to limit LN metastasis and significantly prolong overall survival, anti-VEGFR3 failed to show therapeutic efficacy when given in a similar regimen (Fig. 6J; Supplementary Fig. S28A and S28B). Accordingly, we observed a clear reduction of intratumoral lymphatics only upon short-term Ang2 but not upon short-term VEGFR3 inhibition (Fig. 6K and L; Supplementary Fig. S28C and S28D). In summary, the data demonstrate that acute VEGFR3 blockade is ineffective in targeting an established intratumoral lymphatic network, whereas anti-Ang2 therapy, even when applied just in a brief presurgical regimen, has therapeutic potential by inducing lymphatic regression which translates into suppression of lymphogenous metastasis.

\section{DISCUSSION}

Metastatic growth in the sentinel LN is a well-established prognostic indicator for most cancers (3). In contrast, the clinical relevance of a lymphogenous path of cancer cell dissemination remains highly debated, in part owing to recent melanoma and mammary carcinoma trials wherein locoregional LA failed to improve overall survival $(13,14)$. Yet, the latest genetic data from patients with melanoma, prostate cancer, and colorectal cancer have laid a solid foundation for the notion that at least a portion of peripheral metastases arise from previously established LN metastases, instead of directly originating from the primary tumor $(10,53,54)$. Here, we developed a unique GEMM-derived allograft mouse model of MM that preferentially metastasizes via a sequential lymphogenous route, a fact we could experimentally demonstrate via selective inhibition of lymphatic but not hematogenous dissemination by chemical and surgical approaches. Elegant preclinical work had previously demonstrated the general capacity of cancer cells to spread from LN toward distant organs $(11,12)$. However, to our knowledge, this is the first report of a spontaneous metastasis mouse model in which lymphogenous spread is rate-limiting for peripheral metastasis formation and long-term survival, thereby offering a rare opportunity to study the efficacy of dissemination route-specific therapies.

Employing sophisticated genetic targeting in murine breast cancer models, the pivotal role of tumor-associated lymphatics for the spread of cancer cells to LN and beyond was only recently demonstrated (6). Moreover, lymph fluid was recently identified as a metabolically favorable environment for disseminated melanoma cells (55). Concomitantly, the metastatic potential of the MT-ret model seemed to be dependent on a dense intratumoral network of functional (i.e., draining) lymphatic capillaries. Although LN metastasis can in principle occur in the absence of intratumoral lymphatics (most likely via tumor-surrounding peripheral vessels; ref. 17), it is reasonable to assume that a functional intratumoral lymphatic system accelerates lymphogenous dissemination, especially in the presence of CCL21/CCR7driven tumor cell chemotaxis $(43,44)$. In our modeling approach, we achieved preservation of intratumoral lymphatic vessels by direct orthotopic implantation of tumor fragments instead of a GEMM-derived cell line. This is compatible with the observation that newer patient-derived xenografts often contain functional lymphatics, whereas they are dysfunctional or largely absent in most traditional cell linebased xenograft models (56). Lymphatic capillaries require anchoring filaments, connecting LEC to the surrounding

Figure 5. Intratumoral lymphatic EC require agonistic Ang2-Tie2 signaling. A-D, 10xGenomics of EC-enriched single cells of dissociated MT-ret tumors (pooled from 10 mice). A, Gene signatures of LEC, BEC, and contaminating cell populations based on the relative expression levels of the 10 most-upregulated genes for each of the identified 12 single-cell clusters. Fifty randomly selected cells per cluster are displayed. B, t-SNE visualization of color-coded cell clustering ( $n=7,635$ cells). Dashed lines encompass clusters of different cell types and CEC. C, Dot plot analysis of 5 marker genes for each major cell subpopulation. Dot size reflects proportion of cells of each subpopulation expressing the marker gene. Dot color reflects average marker gene expression. D, Feature plots indicating expression levels of genes associated with the Ang-Tie signaling pathway. E-G, LEC-specific Tie2 deletion after MT-ret fragment transplantation in Tie2 ${ }^{\text {iLECKO }}$ mice. E, Representative CD31/Lyve1 stained tumor sections. F, Relative quantification of Lyve1+ lymphatic area. Data are shown as mean \pm SD, normalized to the Tie2 ${ }^{\text {WT }}$ average. G, Kaplan-Meier survival analysis upon lymphendothelial Tie2 deletion. Scale bars, $200 \mu \mathrm{m}$. Statistical analyses were performed using Mann-Whitney $U(\mathbf{F})$ and Log-rank $(\mathbf{G})$ tests: ${ }^{* \mathrm{k}_{\mathrm{*}} \mathrm{k}}, P<0.001$. 
A

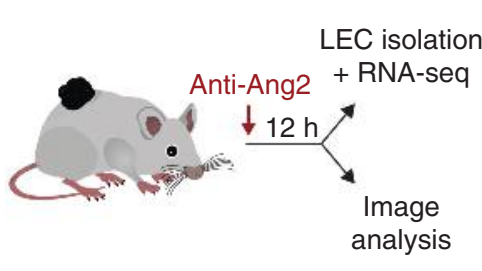

B

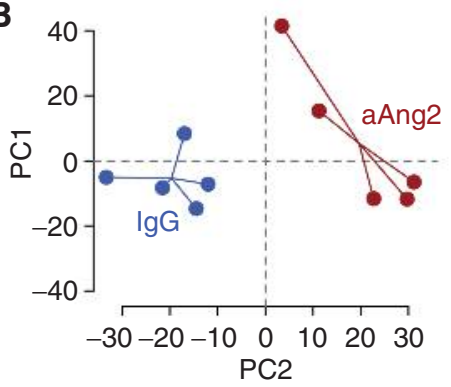

C

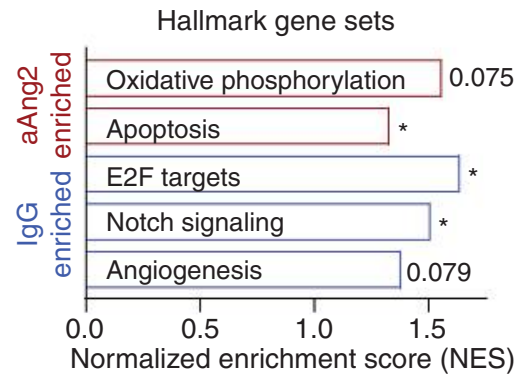

D

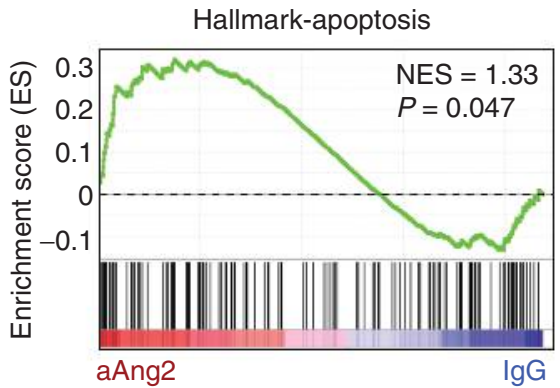

F

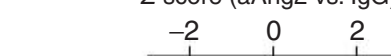

MIF-mediated glucocofticoid regulation (2.29)

VDR/RXR activation (1.32)

Apoptosis signaling (4.62)

PTEN signaling (4.08)

G2/M DNA damage checkpoint regulation (2.07)

VEGF signaling (2.63)

Cell cycle regulation by BTG family proteins (1.65)

NFKB signaling (6.89)

Angiopoietin signaling (2.50)

Estrogen-mediated S-phase entry (2.41)
E

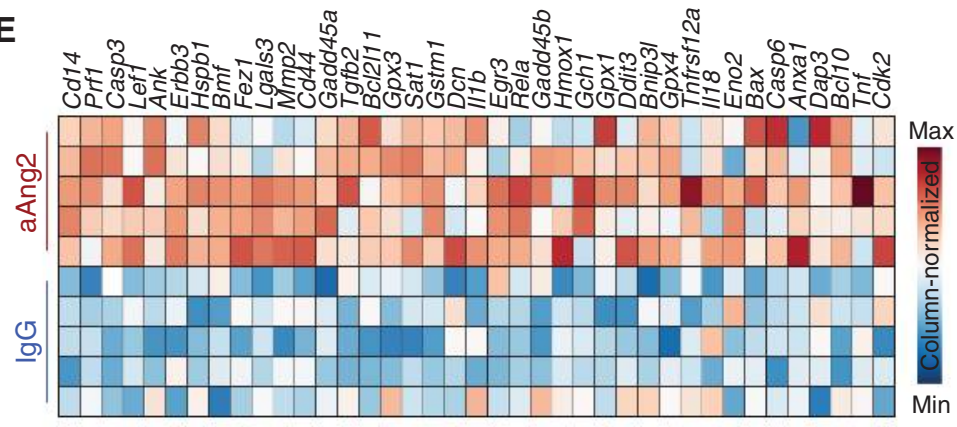

I

Presurgical pulse therapy

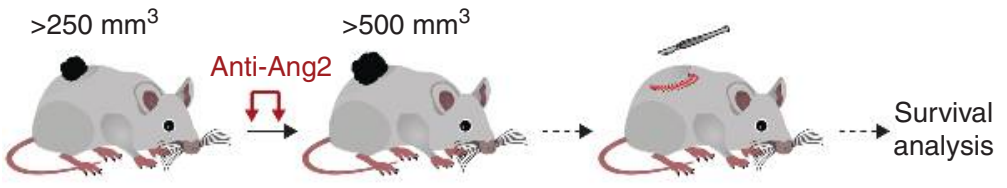

K

$\lg G$

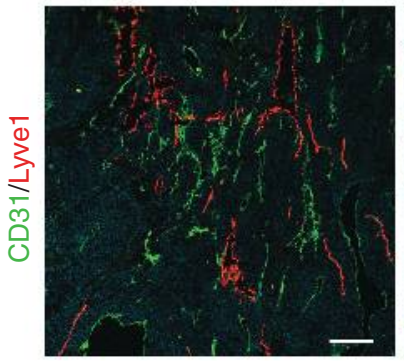

Anti-Ang2

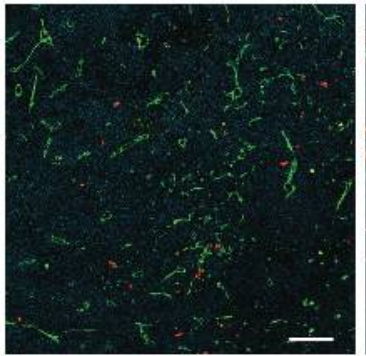

G

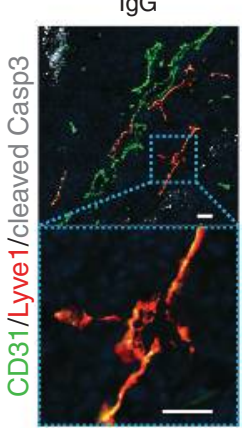

Anti-Ang2

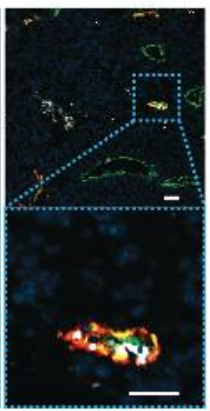

H

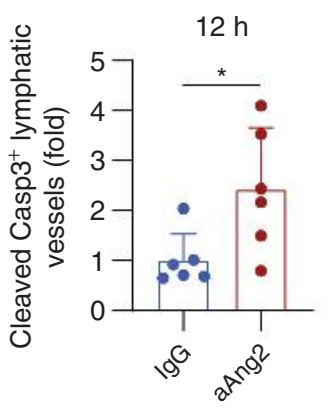

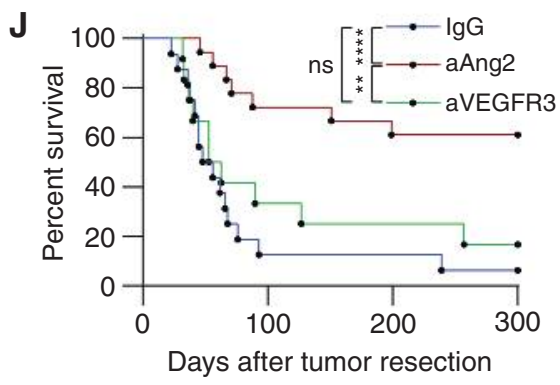

Anti-VEGFR3

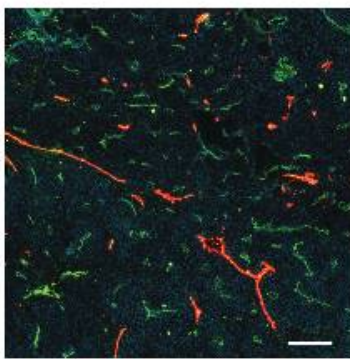

L

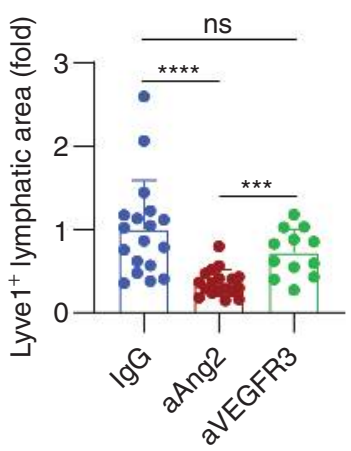


extracellular matrix (ECM), to prevent their collapse due to high interstitial pressure (57). It is therefore tempting to hypothesize that the more organized tissue architecture, which can be observed upon fragment instead of cell line transplantation, facilitates proper construction of such ECM filaments and, thus, the establishment of a fully functional tumor-associated lymphatic system.

Molecular features of the blood vascular tumor endothelium have been thoroughly studied (58). In contrast, surprisingly few gene-expression profiles of intratumoral LEC have been reported, and these studies either involved in vitro cell expansion or included cells from peritumoral nonmalignant tissue, both reflecting the technical hurdle to purify sufficient numbers of viable LEC from tumors $(59,60)$. The abundance of lymphatics in the MT-ret model allowed pure isolation of intratumoral LEC and enabled us to contrast transcriptomic signatures of LEC and BEC via single-cell RNA-seq. Next to the confirmation of a previously reported tumor BEC heterogeneity along a stalk-like to tip-like cell axis $(45,61)$, we provide here first evidence for substantial transcriptomic diversity within the intratumoral LEC compartment. This is in line with recent human and mouse single-cell studies that defined distinct subtypes of LN-associated LEC (62-64). One noteworthy intratumoral LEC cluster, which we identified, displayed marker gene expression resembling a recently described CD74- and MHC class II-expressing subset of cancer-associated fibroblasts (65), hence indicating that such potentially "antigenpresenting" subpopulations might exist within multiple stromal compartments in tumors. Moreover, the transcriptomic profile of this particular LEC cluster was reminiscent of IFN $\gamma$-activated dermal lymphatics that have been described to suppress cytotoxic T cells (66), which is further consistent with a number of recent landmark studies suggesting immune-modulatory LEC function (67-70).

The primarily EC-specific Angiopoietin-Tie system has been extensively described for its key role in blood vascular and lymphatic development and mainly consists of the agonistic ligand Ang1, the partial-agonist Ang2, and the receptor tyrosine kinases Tie1 and Tie2 (22). Single-cell data of this pathway revealed a striking tumor EC-type distinctive pattern especially for Tie2-expressing EC. These were restricted to stalk-like BEC, but rather homogenously distributed over all LEC clusters, suggesting that Tie2 signaling might be negligible for a subset of BEC, whereas possibly essential for all intratumoral LEC. Accordingly, low Tie2 expression has been described for blood vascular endothelial tip cells during spouting angiogenesis, whereas similar in vivo findings are missing in a lymphangiogenic context $(46,47)$. Furthermore, a recent publication reported abrogation of developmental lymphangiogenesis upon genetic Prox1-Cre ${ }^{\text {ERT2 }}$-driven Tie2 deletion between embryonic days 10.5 and 14.5, resulting in severe subcutaneous edema formation (48). By contrast, Shen and colleagues (using an inducible global Tie2 knockout model) had previously demonstrated that postnatal Tie2 is dispensable for lymphatic function (71). Similarly, Ang2 neutralization has been shown to inhibit lymphatic remodeling and valve formation during embryogenesis, but to elicit no apparent phenotype in normal tissue lymphatics of adult mice (72). We found that both LEC-specific Tie2 deletion and Ang2 inhibition during primary tumor growth disrupted the intratumoral lymphatic system, indicating that tumorassociated lymphatics exhibit an Ang2- and Tie2-dependent and, thus, more "developmental" phenotype. Conversely, nontumor lymphatics seemed to be largely unaffected by Tie2 deletion or anti-Ang2 therapy, because we did not observe any edema formation, besides in peritumoral regions (data not shown), altogether implicating the Ang2-Tie2 signaling axis as a potential molecular target to selectively affect the tumorassociated lymphatic system.

In context of blood vessels, Ang2, which is released by EC themselves, usually inhibits Ang1-Tie2 signaling, leading to vascular destabilization and induction of angiogenesis (73, 74). However, in LEC, Ang2 is believed to predominantly act as a Tie2 agonist, partially owing to the observation that developmental lymphatic defects in Ang2 knockout mice can be rescued by ectopic overexpression of the obligatory Tie2 agonist Ang1 (23, 24, 72). Recently, absence of VE-PTP in LEC has been shown to enforce agonistic Tie 2 response toward Ang2 (48). Correspondingly, we observed weak Ptprb expression in all intratumoral LEC compared with BEC. In addition, genetic and antibody-mediated Ang2 inhibition phenocopied LEC-specific Tie2 deletion in terms of intratumoral lymphatic defects, thus also substantiating an agonistic role of Ang2 for the tumor-associated lymphendothelium.

Increased expression of Ang2 has been associated with a wide range of cancer types including melanoma, pancreatic ductal adenocarcinoma (PDAC), renal cell carcinoma, glioblastoma, breast carcinoma, and colorectal carcinoma, thus rendering it an excellent angiogenic candidate molecule for therapeutic intervention $(26,75-78)$. Consequently, diverse Ang2-targeted therapies are in clinical development (25). Most preclinical studies addressing Ang2 neutralization in tumor progression and metastasis have mechanistically focused on the tumor-associated blood vascular system. Accordingly, Ang2 inhibition was reported to delay primary tumor growth by decreasing angiogenic sprouting and by inducing vascular

Figure 6. Acute Ang2 blockade drives lymphatic regression and prolongs survival. A, Schematic overview outlining the experimental strategy for a short-term Ang2 inhibition in the MT-ret model. B, Principal component analysis of bulk RNA-seq data of isolated tumor LEC upon short-term Ang2 inhibition. C, GSEA of hallmark gene sets. P values are indicated. D, Enrichment plot of the apoptosis hallmark gene set. E, Heat map highlighting all significantly enriched genes within the apoptosis gene set. $\mathbf{F}$, Ingenuity canonical pathways analysis of all differentially regulated genes. Brackets indicate - log ${ }_{10}\left(P_{\text {adj }}\right)$ G, CD31/Lyve1/cleaved Casp3 stained tumor sections. H, Relative quantification of cleaved Casp3+ lymphatic vessels. I, Schematic overview depicting the strategy of a presurgical pulse therapy in the MT-ret model. J, Kaplan-Meier survival analysis upon acute Ang2 or VEGFR3 blockade. K, Representative CD31/Lyve1 stained tumor sections. L, Relative quantification of Lyve1+ lymphatic area. Scale bars, $50 \mu \mathrm{m}$ (G) and $200 \mu \mathrm{m}(\mathbf{K})$. Data are shown as mean \pm SD, normalized to the IgG average $(\mathbf{H}$ and $\mathbf{L})$. Statistical analyses were performed using Mann-Whitney $U(\mathbf{H}$ and $\mathbf{L})$ and $\mathbf{L}$ og-rank $(\mathbf{J})$ tests. ns, not signifi-

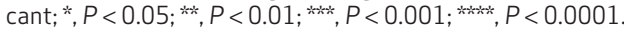


normalization, thus acting in synergy with VEGF blockade and chemotherapy $(28-30,79)$. In addition, Ang2-blocking antibodies were shown to stabilize the blood vasculature at sites of cancer cell intravasation and extravasation, to interfere with macrophage function, and to inhibit premetastatic niche preparation, all impeding metastasis formation $(27,80$, 81 . By contrast, only a few scattered reports have hitherto addressed the tumor-associated lymphatic system. In those, ectopic Ang2 overexpression in PDAC and lung carcinoma xenografts resulted in enhanced LN metastasis. Furthermore, an Ang2-blocking antibody was described to suppress tumor lymphangiogenesis in a lung cancer xenograft model $(27,82)$. Here, employing multiple syngeneic mouse tumor models, we report more pronounced lymphatic than blood vascular effects of antibody-mediated Ang2 neutralization, leading to an efficient blockade of lymphogenously seeded fatal metastasis. Although tumor experiments in Ang $2^{\mathrm{KO}}$ mice similarly revealed decreased intratumoral lymphatics, disrupted tumor drainage, impeded metastasis, and prolonged survival, it should be noted that these data cannot strictly be interpreted as active targeting of intratumoral lymphatic vessels, because Ang $2^{\mathrm{KO}}$ mice suffer from baseline lymphatic defects due to impaired lymphatic maturation during embryonic development (23). Ang2 had been previously described to predominantly affect early stages of primary tumor growth but to be largely dispensable for the growth of established tumors (40). Similarly, we found tumor blood vessels to be unaffected by short-term presurgical Ang2 neutralization (i.e., in mice carrying large tumors). In sharp contrast, acute Ang2 inhibition was sufficient to regress even an established intratumoral lymphatic network, most likely by interfering with Tie2-dependent survival cues. Corroborating published literature, anti-VEGFR3 therapy effectively inhibited tumor lymphangiogenesis in a long-term treatment regimen (3-5). Yet, contrary to Ang2 neutralization, acute VEGFR3 blockade had no major impact on already-established lymphatics, suggesting that lymphatic maintenance did not rely on VEGFR3 signaling. It remains to be resolved if the strong dependency of intratumoral lymphatics on Ang2 (opposed to VEGFC/VEGFD) would vary in tumor patients and models with a diverging growth factor availability.

As an experimental tool, primary tumor resection allowed us to model therapeutic Ang2 neutralization in diverse treatment regimens. Fitting to our hypothesis of a predominant role of Ang2 in lymphogenous tumor cell dissemination, only presurgical neoadjuvant but not postsurgical adjuvant Ang2 blockade yielded a robust and longlasting survival advantage. This was further supported by correlative human melanoma data contrasting primary tumor- and metastasis-derived Ang2, and is in agreement with a number of recent clinical trials, wherein Ang2targeted drugs showed unsatisfactory results when administered to patients with relapsed or metastasized ovarian and colorectal carcinoma, i.e., at a time when tumor cell seeding had already occurred (31-34). Clinical observations in patients with breast cancer have implicated tumor surgery itself as a prominent driver of cancer cell displacement via the lymphatic system, suggesting that late presurgical blockade of lymphogenous seeding might still have therapeutic potential (83). Accordingly, we demonstrated in this study efficacy of Ang2 inhibition to block metastasis, even when administered in the form of an acute treatment pulse only shortly before primary tumor resection, which might be a realistic therapeutic regimen for clinical use. Moreover, in such a therapeutic window, the extravasation-blocking efficacy of anti-Ang2, shown here and by Holopainen and colleagues (27), likely synergizes with lymphatics-targeted effects of Ang2 neutralization.

In conclusion, employing multiple preclinical mouse tumor models including a unique novel GEMM-derived allograft fragment transplantation system in combination with surgical primary tumor removal, we identified presurgical Ang2 neutralization as an efficient lymphatics-targeted migrastatic therapy (84). The study has thereby shed fundamental insight into the key role of the Angiopoietin-Tie signaling pathway in controlling regional and distant lymphatic metastasis. Further supported by the human biomarker data presented in this study, future experimental and clinical work is warranted to translate these findings into human cancer, potentially by identifying patient subpopulations that are still node-negative but at risk of lymphogenous tumor cell dissemination.

\section{METHODS \\ Cells}

All cells were cultured at $37^{\circ} \mathrm{C}$ and $5 \% \mathrm{CO}_{2}$ and were routinely tested for Mycoplasma by PCR (Primers: $5^{\prime}$-TGCACCATCTGTCAC TCTGTTAACCTC and 5'-GGGAGCAAACAGGATTAGATACCCT). LLC (ATCC), B16F10 Luc2 (Caliper Life Sciences), and MT-ret cells (established from a spontaneously developed tumor in MT-ret transgenic mice) were cultured in DMEM GlutaMAX (Thermo Fisher Scientific). MT-ret GFP cells were established by lentiviral transduction of a TurboGFP reporter (Dharmacon, RHS4346). 4T1-Luc cells (kindly provided by G. Sahagian, Tufts University, MA) were cultured in RPMI 1640 (Thermo Fisher Scientific). DMEM GlutaMAX and RPMI 1640 were supplemented with $10 \%$ FCS and 1\% penicillin/streptomycin (Sigma-Aldrich). Primary HDLEC (PromoCell, C-12216) were used between passages two and six and cultured on $0.2 \%$ gelatin-coated plates in Endothelial Cell Growth Medium MV2 (PromoCell), supplemented with the corresponding supplement mix and $1 \%$ penicillin/streptomycin.

\section{Patient Samples}

Archived paraffin-embedded tissue samples from the University Medical Center Mannheim (melanoma tissue microarray) and from the Institute of Pathology, Technical University Munich (melanoma and breast cancer samples), were used. Studies were approved by the ethical committees of Heidelberg University (2010-318N-MA) and Technical University Munich (160/19 S).

\section{Mice}

Female C57BL/6N, SCID, and NSG mice (8-10 weeks old) were purchased from Charles River. MT-ret transgenic mice (C57BL/6 background) were provided by M. Kato (Chubu University, Aichi, Japan) and V. Umansky (UMM, Mannheim, Germany; ref. 35). Tyr$\mathrm{Cr} \mathrm{ERT}^{\mathrm{ER} 2} \times \mathrm{Braf}^{\mathrm{V} 600 \mathrm{E} /+} \times \mathrm{Pten}^{\mathrm{H} / \mathrm{l}} \times \mathrm{Ctnnb}^{\mathrm{E} \times 3 \mathrm{H} / /+}$ mice were provided by M. Bosenberg (Yale University School of Medicine, New Haven, CT; ref. 85). mTmG mice (C57BL/6 background) were provided by $\mathrm{P}$. Angel (DKFZ, Heidelberg, Germany; ref. 86). Ang $2^{\mathrm{KO}}$ mice were bred into the C57BL/ 6 background and maintained by heterozygous mating (40). Homozygous Ang $2^{\mathrm{KO}}$ and Ang2 ${ }^{\mathrm{WT}}$ littermates were used for experiments. Prox1-Cre $e^{\mathrm{ERT} 2}$ mice were provided by G. Oliver 
(Northwestern Medicine). Prox1-Cre $e^{\mathrm{ERT} 2+/-} \times \mathrm{Tie}^{\mathrm{f} / \mathrm{l}}$ mice were bred with Cre-negative Tie $2^{\mathrm{f} / \mathrm{l}}$ mice (87) to generate $50 \%$ Cre-positive (Prox1-Cre ${ }^{\mathrm{ERT} 2+/-} \times \mathrm{Tie}^{\mathrm{H} / \mathrm{f}}$ mice, termed Tie $2^{\mathrm{iLECKO}}$ ) and $50 \%$ Cre-negative (Tie $2^{\mathrm{f} / \mathrm{fl}}$ mice, termed Tie $2^{\mathrm{WT}}$ ) littermates. Eight- to 12 -week-old male and female transgenic mice were used for experiments. All mice were housed on a 12-hour-light/-dark cycle with free access to food and drinking water in specific pathogen-free animal facilities. All experiments were performed according to the guidelines of the institutional and governmental Animal Care and Use Committees and approved by the Regierungspräsidium Karlsruhe (DKFZ305, DKFZ370, G9-19, G257-18, G255-18, G254-18, G231-16, and G244-14).

\section{Tumor Models and Pharmacologic Interventions}

All mice were routinely checked for clinical endpoint criteria. Tumor volumes were determined by caliper measurements $(3 \times$ per week; tumor volume $=1 / 2$ length $\times$ width $\times$ height). In antibody-mediated neutralization experiments, mice were treated every 3 days by i.p. injection of anti-Ang2 $(20 \mathrm{mg} / \mathrm{kg}, 18 \mathrm{E} 5$, Eli Lilly and Company), antiVEGFR2 (20 mg/kg, DC101, Eli Lilly and Company), anti-VEGFR3 (30 $\mathrm{mg} / \mathrm{kg}, 31 \mathrm{C} 1$, Eli Lilly and Company), or corresponding mouse (Eli Lilly and Company) and rat (BioXcell) IgG1 isotype controls. In survival studies, gross necropsy was performed on all moribund animals or at indicated experimental endpoints. Survival times were defined as the number of days after primary tumor resection until the first clinical endpoint was reached. Endpoints consisted of (i) metastasis with a diameter of $>1.5 \mathrm{~cm}$, (ii) weight loss of $20 \%$ (compared with normal weight), (iii) lack of feed or water intake, (iv) noticeable respiratory symptoms, (v) abnormal posture or crouching, (vi) apathy or immobility, and (vii) pale extremities.

\section{MT-ret Melanoma Fragment Transplantation Model (MT-ret Model)}

Model Establishment. Spontaneous tumor nodules of 7- to 12 -week-old MT-ret mice were harvested, and tumor fragments $(\varnothing=$ $2-3 \mathrm{~mm}$ ) were directly implanted into the dermis of C57BL $/ 6 \mathrm{~N}$ mice (abdominal skin, midline region). Upon successful engraftment, MT-ret allografts were biobanked at $-196^{\circ} \mathrm{C}$ in DMEM/F12, HEPES (Thermo Fisher Scientific), supplemented with 10\% FCS and 10\% DMSO, and serially retransplanted for in vivo expansion. Fragments of the third to fifth passages were used for all experiments.

Experimental Use. Biobanked MT-ret tumor fragments $(\varnothing=2-3$ $\mathrm{mm}$ ) were orthotopically implanted into C57BL/6N, NSG, Ang $2^{\mathrm{KO} / \mathrm{WT}}$, or $\mathrm{Tie} 2^{\mathrm{iLECKO} / \mathrm{WT}}$ mice. All primary tumors were surgically resected when their size surpassed a cutoff volume of $500 \mathrm{~mm}^{3}$. Neoadjuvant antibody treatment was initiated 7 days after fragment implantation and discontinued after primary tumor resection. Adjuvant treatment was started 4 days after primary tumor resection and continued throughout the experiment. In presurgical pulse experiments, two doses of antibody were given when primary tumors surpassed a cutoff volume of $250 \mathrm{~mm}^{3}$. To induce lymphendothelial-specific Tie2 deletion, Tie $2^{\text {iLECKO }}$ and control animals were i.p. injected $2 \times$ per week with tamoxifen (Sigma-Aldrich, $2 \mathrm{mg}$ dissolved in ethanol and peanut oil). Tamoxifen treatment was started at time of fragment implantation and stopped after primary tumor resection.

\section{TBPC Melanoma Model}

Focal melanoma induction on the ventral flank of $\mathrm{Ty} r$-Cre $e^{\mathrm{ERT}} \times$ $\mathrm{Braf}^{\mathrm{y} 600 \mathrm{E} /+} \times \mathrm{Pten}^{\mathrm{H} / \mathrm{f}} \times \mathrm{Ctnnb1}^{\mathrm{E} \times 3 \mathrm{H} / /+}$ mice was performed by intradermal

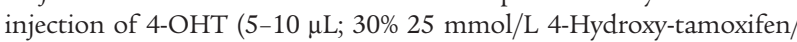
$70 \%$ polyethylene glycol; Sigma-Aldrich). Every other day, mice were individually checked for a palpable tumor elevation. At first detection, antibody treatment was initiated and continued until the tumor size surpassed a cutoff volume of $400 \mathrm{~mm}^{3}$.

\section{LLC Lung Cancer Model}

LLC cells $\left(1 \times 10^{6}\right.$ in PBS) were s.c. inoculated in the ventral flank region of $\mathrm{C} 57 \mathrm{BL} / 6 \mathrm{~N}$ mice. Primary tumors were surgically resected after 14 days of tumor growth. Antibody treatment was initiated 4 days after LLC injection and stopped at time of primary tumor resection.

\section{T1-Luc Breast Cancer Model}

4T1-Luc cells $\left(5 \times 10^{5}\right.$ in PBS) were orthotopically implanted in the fourth right mammary fat pad of SCID mice. Mastectomy was performed after 21 days of tumor growth. Antibody treatment was initiated 4 days after 4T1-Luc injection and stopped at time of primary tumor resection. Fourteen days after mastectomy, metastasis formation was quantified by organ-specific ex vivo bioluminescence imaging (BLI) measurements.

\section{MT-ret Cell Line-Based/MT-ret GFP Melanoma Model}

MT-ret cells or MT-ret GFP cells $\left(1 \times 10^{6}\right.$ in PBS) were intradermally inoculated in the ventral flank region of C57BL/6N mice.

\section{In Vivo Imaging}

BLI quantification of 4T1-Luc or B16F10 Luc2 metastasis was carried out on an IVIS-200 system (PerkinElmer). Mice were i.p. injected with $150 \mathrm{mg} / \mathrm{kg}$ RediJect D-luciferin (PerkinElmer) 7 minutes prior to imaging. Afterward, mice were anesthetized with isoflurane, and luminescence measurements were acquired according to manufacturer's instructions. Signal quantification was performed with constant size region of interests (ROI) in the Living Image software 4.0 (PerkinElmer). In the case of ex vivo analysis, animals were sacrificed 5 minutes after injection of $150 \mathrm{mg} / \mathrm{kg}$ RediJect D-luciferin. Tissues were dissected and placed in 6-well plates for organ-specific luminescence measurements.

$\mathrm{F}^{18}$-FDG-PET/CT imaging was performed by the DKFZ Small Animal Imaging Core facility using the Inveon three-modality small animal scanner (Siemens).

\section{Tumor Drainage}

Evans Blue Drainage. Evans blue dye $(10 \mu \mathrm{L} ; 2 \%$ in PBS, SigmaAldrich) was injected into the tumors of ketamine/xylazine-anesthetized mice using a Hamilton syringe. Ten minutes later, inguinal and axillary $\mathrm{LN}$ were isolated, imaged under a stereomicroscope, and dried for 24 hours at $80^{\circ} \mathrm{C}$. Evans blue was subsequently extracted from the $\mathrm{LN}$ by overnight incubation in formamide at $55^{\circ} \mathrm{C}$ and quantified by measuring the absorbance at a wavelength of $620 \mathrm{~nm}$. Data were normalized to the LN dry weight.

Rhodamine Dextran Drainage. High-molecular-weightrhodamine dextran $(10 \mu \mathrm{L} ; 2,000 \mathrm{kDa}, 10 \mathrm{mg} / \mathrm{mL}$, Thermo Fisher Scientific) was injected into the tumor of ketamine/xylazine-anesthetized mice using a Hamilton syringe. Thirty minutes later, inguinal and axillary LNs were isolated and embedded in Tissue-Tek OCT compound (Sakura). LNs were cut in $10 \mu \mathrm{m}$ sections and stained for Lyve 1 and with Hoechst. LN sections were imaged with an Axio Scan.Z1 slide scanner (Zeiss) and subsequently analyzed in Fiji. Rhodamine-positive area was quantified relative to the total $\mathrm{LN}$ area. All values were normalized to the average of control samples.

\section{Lymphadenectomy}

LA of all inguinal and axillary LN was performed in ketamine/ xylazine-anesthetized mice according to an adapted murine superficial LN surgery protocol (88). To limit surgical discomfort, only two LNs were removed at the same time. In case of protective LA, both axillary LNs were removed 2 days prior to and both inguinal LN at time of primary tumor implantation. In the case of therapeutic LA, both inguinal LNs were removed at time of, and both axillary LN 2 days after, primary tumor resection. 


\section{Experimental Metastasis Assay}

C57BL/6N mice were pretreated with anti-Ang2 or IgG, 4 and 1 days prior to tumor cell injection. $2 \times 10^{5}$ B16F10 Luc2 or MT-ret GFP cells (in PBS) were injected into the tail vein. Lungs were collected 2 weeks after tumor cell inoculation, and metastatic foci were counted under a stereomicroscope and quantified by flow cytometry of BLI imaging.

\section{Ex Vivo Culture of MT-ret Circulating Tumor Cells}

Arterial blood was isolated by cardiac puncture and kept in Microtainer tubes (lithium heparin; BD Biosciences). One hundred microliters blood was plated in $2 \mathrm{~mL}$ DMEM plus $10 \%$ FCS and 1\% penicillin/ streptomycin in 12-well plates, and 14 days later, pigmented tumor cell colonies were counted under an Olympus IX71 microscope. Because circulating tumor cell numbers have been described to correlate with tumor size (89), data were normalized to the tumor volume at time of blood collection.

\section{Retina Angiogenesis Assay}

Newborn pups were i.p. administered with anti-Ang2 or IgG on postnatal days 1 and 4 . On postnatal day 6 , eyeballs were isolated and fixed in methanol at $-20^{\circ} \mathrm{C}$. Blocking and permeabilization were performed with $10 \%$ normal goat serum $/ 0.5 \%$ Triton-X100/1\% BSA in PBS for 1 hour at room temperature (RT). The retinal vasculature was stained by incubation with Isolectin B4 (Sigma-Aldrich, 1:100) and a NG2 primary antibody (Sigma-Aldrich, \#Ab5320; 1:1,000) diluted in $0.2 \%$ Tween-20/1\% BSA in PBS overnight at $4{ }^{\circ} \mathrm{C}$, followed by incubation with appropriate secondary antibodies. After each antibody incubation, retinas were washed 3 times for 5 minutes each with $0.2 \%$ Tween- 20 in PBS. Fluorescently labeled retinas were flatmounted with DAKO mounting medium and imaged using the Zeiss LSM780 microscope with a 20x objective. Image analysis was done in Fiji. Vessel area was calculated as Isolectin B4-positive area per retina area. Vessel outgrowth reflects the average distance between the optic nerve and the outer rim of a vascularized retina leaf. Pericyte coverage was calculated as NG2-positive area per vessel area. All values were normalized to the average of control-treated pups.

\section{Tissue Staining}

Immunofluorescence. Tumors and organs were embedded in Tissue-Tek OCT compound (Sakura) and cut into 7 to $10 \mu \mathrm{m}$ sections. Cryosections were fixed and permeabilized in ice-cold methanol for 10 minutes at $-20^{\circ} \mathrm{C}$. Following washing, sections were blocked in $10 \%$ ready-to-use normal goat serum (Thermo Fisher Scientific) or $10 \%$ donkey serum (Thermo Fisher Scientific) for 1 hour at RT, followed by overnight incubation with primary antibodies at $4^{\circ} \mathrm{C}$. Incubation with corresponding secondary antibodies and fluorescent dye-conjugated primary antibodies was performed the next day for 1 hour at RT. Cell nuclei were counterstained with Hoechst (SigmaAldrich), and sections were mounted with DAKO mounting medium (Agilent). Images were taken as whole-area tile scans using an Axio Scan.Z1 slide scanner (Zeiss), and image analysis was performed in Fiji using automated thresholding techniques. CD31-, Lyve1-, and VEGFR3-positive (lymphatic-) vessel areas were normalized to the total tissue area. Pericyte coverage was determined by normalizing vessel-associated Desmin- or aSMA-positive area to the total CD31positive area. Lymphatic regression was determined by colocalizing the lymphatic area with the cleaved Casp3- or PARP1-positive area. If not indicated otherwise, all values were normalized to the average of control samples, thereby allowing the comparison of independent experiments.

Primary AB. CD31 (MEC13.3), Desmin (Abcam; Ab15200-1), aSMA (14A), cleaved Caspase-3 (Cell Signaling Technology, \#9661), cleaved PARP1 (Cell Signaling Technology, \#5625), Lyve1 (Reliatech, 103-PA50AG), VEGFR3 (R\&D Systems, AF743), CD45 (30-F11), CD3 (Dako, A045229-2), and CD11b (M1/70) were used.

The Alexa Fluor 647 Antibody Labeling Kit (Thermo Fisher Scientific) was used for direct labeling of primary antibodies.

Secondary AB. Goat anti-rat Alexa 488, goat anti-rabbit Alexa 647, goat anti-rabbit Alexa 546, and donkey anti-rabbit Alexa 647 (Thermo Fisher Scientific) were used.

Immunohistochemistry. Zinc-fixed murine and formalin-fixed human samples were used. Following paraffin embedding, samples were cut into $7 \mu \mathrm{m}$ sections, dewaxed, and rehydrated. Images were taken as whole-area tile scans using an Axio Scan.Z1 slide scanner (Zeiss).

For necrosis analysis, sections were stained with hematoxylin and eosin (H\&E) and analyzed in a blinded manner by a board-certified pathologist (C. Mogler, TUM, Munich, Germany) to assess percentage of necrotic area per tumor.

For Lyve1 (Reliatech, 103-PA50AG) immunohistochemistry, antigen unmasking was performed using citrate buffer $(\mathrm{pH} 6)$ at $95^{\circ} \mathrm{C}$ for 20 minutes. To inactivate endogenous peroxidase activity, slides were subjected to 15 minutes of $3 \% \mathrm{H}_{2} \mathrm{O}_{2}$. Sections were subsequently blocked in 3\% BSA $/ 10 \%$ normal goat serum for 1 hour at RT. Primary antibody incubation was performed overnight at $4^{\circ} \mathrm{C}$, followed by 30-minute incubation with a secondary goat anti-rabbit biotinylated. Antibody detection was visualized by DAB after ABC-HRP incubation (Vector Laboratories).

Serial sections of human samples were stained for Ang2 (Fitzgerald Industries, 20R-AR043) and Podoplanin (D2-40) at the Institute of Pathology (Heidelberg University) using an automated IHC stainer (Ventana) in accordance with the manufacturer's protocol. All biopsies from Ang2-stained melanoma tissue microarrays were stratified according to their anatomic origin from a primary tumor or a metastatic lesion. Complementary patient survival data were kindly provided by J. Utikal (UMM, Mannheim, Germany). Scoring was performed in a blinded manner by a board-certified pathologist (C. Mogler, TUM, Munich, Germany). A scoring scheme from 0 to 3 was used: 0 (no expression), 1 (weak expression), 2 (moderate expression), and 3 (high expression). For analysis of Supplementary Fig. S16, vascular D2-40 signals, which were either located within a demarcated primary tumor mass or closely associated with invasive tumor cell islands, were considered as tumor-associated lymphatics and taken into account for scoring.

\section{Flow Cytometry}

To reduce nonspecific binding to cells bearing Fc receptors, preincubation of cells with anti-mouse CD16/CD32 was performed prior to all antibody stainings. Dead cells were excluded by FxCycle Violet (Thermo Fisher Scientific) staining. Stained cells were analyzed and FACS-sorted using a BD bioscience Aria cell sorting platform, and frequencies of individual cell populations were quantified with FlowJo software.

Cell Isolations. Tumors were dissociated with Liberase digestion enzyme mix (Roche) at $37^{\circ} \mathrm{C}$ for 30 minutes. Single-cell suspensions were prepared by passing digested tissues through 19G cannula syringes and filtering through a $100 \mu \mathrm{m}$ cell strainer. Red blood cells (RBC) were lysed for 5 minutes in $1 \times \mathrm{RBC}$ lysis buffer (Thermo Fisher Scientific). Thereafter, ECs were enriched using CD31 microbeads (Miltenyi Biotec) according to the manufacturer's instructions. Enriched ECs were stained for 45 minutes at $4^{\circ} \mathrm{C}$ using fluorescent dye-conjugated antibodies [CD45 (30-F11), CD31 (MEC13.3), and LYVE1 (ALY7)] and subsequently FACS-sorted for blood vascular EC- and lymphatic EC-specific surface marker profiles.

CCR7 Surface Presentation. MT-ret cells were stained for CD197 (4B12) or with rat IgG2a kappa isotype control for 2 hours at RT. 
FACS-Based Immune Phenotyping. MT-ret tumors were dissociated into single-cell suspensions with Liberase digestion enzyme mix (Roche). Following RBC lysis, the remaining single-cell solutions were split for lymphoid [CD45 (30-F11), CD3ع (17A2), CD4 (GK1.5), CD8a (53-6.7), CD45R-B220 (RA3-6B2), and NK-1.1 (PK136)] and myeloid [CD45 (30-F11), CD11b (M1/70), Ly6C (HK-1.4), Ly6G (1A8), CD11c (N418), CD68 (FA-11), and Tie2 (TEK4)] staining.

Quantification of Circulating or Metastasized MT-ret GFP Cells. For quantification of circulating MT-ret GFP cells, $500 \mu \mathrm{L}$ arterial blood of MT-ret GFP tumor-bearing mice was isolated by cardiac puncture and kept in Microtainer tubes (lithium heparin; BD Biosciences). For quantification of experimental MT-ret GFP lung metastasis, lungs were dissociated with Liberase digestion enzyme mix (Roche) at $37^{\circ} \mathrm{C}$ for 30 minutes. Single-cell suspensions were prepared by passing digested tissues through 19G cannula syringes and filtering through a $100 \mu \mathrm{m}$ cell strainer. Following RBC lysis and dead cell exclusion, GFP-positive tumor cells were quantified using CountBright Absolute Cell Counting Beads (Thermo Fisher Scientific) according to the manufacturer's instructions. Gates for GFP positivity were set according to cultured MT-ret GFP cells. Data were normalized to the tumor volume at time of blood collection.

\section{ELISA}

Ang2, CCL21, and VEGFC protein levels in serum or tumor lysates were determined using the Mouse/Rat Angiopoietin2 Quantikine ELISA Kit (R\&D Systems), the Mouse CCL21/6Ckine Quantikine ELISA Kit (R\&D Systems), and the Mouse Vascular Endothelial Cell Growth Factor C ELISA kit (Cusabio), according to the manufacturers' instructions.

\section{Cytokine Profiling}

Proteome Profiler Mouse XL Cytokine Arrays (R\&D Systems) were performed according to the manufacturer's instructions. A total amount of $250 \mu \mathrm{g}$ protein, pooled from 4 to 6 whole tumor lysates of the same experimental group, was used per membrane. Densitometric quantification with constant size ROI was performed in Fiji.

\section{RNA Extraction and qPCR Analysis}

RNA of FACS-sorted mouse cells was isolated with the Arcturus PicoPure RNA Isolation Kit (Thermo Fisher Scientific) according to the manufacturer's instructions. RNA from whole mouse blood was purified using the Mouse RiboPure-Blood RNA Isolation Kit (Thermo Fisher Scientific) according to the manufacturer's instructions. RNA from tumors or organ tissue was isolated by smashing the tissue pieces in TRIzol (Thermo Fisher Scientific) followed by chloroform extraction. Afterward, RNA was isolated with the RNeasy Mini Kit (Qiagen) according to the manufacturer's instructions. cDNA was synthesized with the QuantiTect Reverse Transcription Kit (Qiagen) according to the manufacturer's instructions. Gene expression analysis was performed by quantitative PCR using TaqMan reactions. The following TaqMan probes (Thermo Fisher Scientific) were used: Actb-Mm00607939_S1; Angpt1-Mm00456503_m1; Angpt2Mm00545822_m1;Angptl4-Mm00480431_m1; Angptl6-Mm00513964_m1; B2M-Hs00984230_m1; Bax-Mm00432051_m1; Bcl2-Mm00477631_m1; Ccl21-Mm03646971_m1; CCL21-Hs00984230_m1; Ccr7-Mm99999130_s1; Cd14-Mm00438094_g1; Dct-Mm01225584_m1; Dll4-Mm00444619_m1; Flt1-Mm00438980_m1; Flt4-Mm01292604_m1; Itgb1-Mm01253230_m1; Kdr-Mm01222421_m1; Lyve1-Mm00475056_m1; Mcl1-Mm01257351_g1; Notch1-Mm00435249_m1; Notch4Mm00440525_m1; Pecam1-Mm01242584_m1; Pgf-Mm00435613_m1; Tek-Mm00443254_m1; Tie1-Mm00441806_m1; Tnfrifla-Mm00441883_g1; Tnfisflb-Mm00441889_m1; Tyr-Mm00495817_m1; Vegfa-Mm01281449_m1; Vegfb-Mm00442102_m1; Vegfc-Mm00437310_ $\mathrm{m} 1$; Vegfd-Mm01131929_m1. Gene expression levels were calculated based on the $\Delta \mathrm{Ct}$ relative quantification method, normalized to Actb expression (90).

\section{Microarray Expression Profiling}

250 ng total tissue RNA, quality-controlled by Bioanalyzer (Agilent) measurements, was used to generate ss-cDNA, which were labeled and hybridized on Affymetrix GeneChip Mouse Gene 2.0 ST arrays (Thermo Fisher Scientific) according to the manufacturer's instructions. Microarray data were quantile normalized with R using the normalize.quantiles function from Bioconductor package "preprocessCore."

\section{Single-Cell RNA-seq}

MT-ret tumor BEC and LEC from ten biological replicates were FACS-sorted and pooled. Cell suspensions, consisting of 10,000 randomly selected BEC and LEC, were separately loaded on a Chromium Single Cell Instrument (10X Genomics). Sequencing libraries were prepared using Chromium Single Cell 3' Reagent Kits (v2 Chemistry). Multiplexed libraries were subsequently sequenced on a HiSeq4000 platform (Illumina).

Data Processing. Single-cell reads were aligned to the mouse reference genome ( $\mathrm{mm} 10)$ using Cell Ranger (version 2.1.1), thereby generating gene-barcode matrices. Low-quality cells, containing fewer than 200 detected genes or with mitochondrial genes accounting for more than $10 \%$ of total transcripts, were filtered out. In individual BEC and LEC samples, outlying cells were further identified via scater $\mathrm{R}$ package (version 1.10.1) using median absolute deviations. Following quality control, BEC and LEC single-cell data were merged and analyzed using Seurat R package (version 2.3.4; refs. 91, 92). All quality-controlled cells $(n=7,635)$ were integrated with Seurat. Highly variable genes were selected based on the dispersion of more than 0.5 and an average expression between 0.0125 and 4 . The number of unique molecular identifier (UMI) varied among cells was regressed out with a linear model.

Unsupervised Clustering and Visualization. We reduced dimensions of data using principal component analysis. First 36 principal components were chosen at the elbow of the curve using PCElbowPlot function. Unsupervised clustering using the FindClusters function (resolution 0.6) yielded 12 distinct clusters. Differentially expressed genes of each cluster were identified with the FindAllMarkers function. For heat-map visualization of cluster defining gene signatures, we randomly subsampled 50 cells from each cluster to neutralize size differences and used the 10 most upregulated genes per cluster. $\mathrm{t}$-SNE was further employed for two-dimensional data visualization.

Biologically Supervised Annotation and Analysis. Unsupervised clusters were manually annotated based on previously known marker gene expression of different cell types and actively cycling cells. For heat-map representation, median gene expression per cluster was calculated with the AverageExpression function. Biologically annotated data were further visualized using DotPlot and FeaturePlot functions. Reducing expression effects of contaminating elements, differentially expressed genes defining specific LEC or BEC subclusters were additionally computed with the FindMarkers function relative only to all other BEC or LEC subclusters, respectively. GO annotation using the g:Profiler web server was performed to identify subclusterspecific molecular pathways and biological functions (93).

\section{Bulk RNA-seq and Data Analysis}

MT-ret tumor LECs were FACS-sorted from five biological replicates of each treatment group, and total RNA was isolated using the Arcturus PicoPure RNA isolation kit (Thermo Fisher Scientific) according to the manufacturer's protocol. Quality control was performed by Bioanalyzer (Agilent) measurements (RNA integrity number $>8.0$ ). Sequencing libraries were generated with $0.5 \mathrm{ng}$ of total RNA using the SMART-Seq v4 Ultra Low Input RNA Kit for Sequencing (SMARTer) and the NEBNext ChIP-Seq Library Prep 
Master Mix Set for Illumina (NEBNext). Sequencing reads (50 bp single read) were generated on the HiSeq4000 platform (Illumina) with five samples per lane. All reads were aligned to the mouse reference genome mm10 using STAR aligner Version 2.5.3a (94). Gene-specific read counts were computed with FeatureCounts and used for subsequent FPKM calculation. Differential gene expression was computed using DEseq2 (95). Only transcripts with an FPKM $\geq 1$ in at least one sample were considered for further downstream analysis. GSEA and IPA were undertaken to study regulated molecular pathways and corresponding biological functions (96).

\section{Statistical Analysis}

Statistical analysis was performed using GraphPad Prism version 8 (GraphPad Software). Unless otherwise specified, data are expressed as mean \pm SD. Statistical tests are indicated in each figure legend. Two-sided tests were employed, unless otherwise specified. A $P$ value of less than 0.05 was considered statistically significant and marked by asterisks ( ${ }^{*}, P<0.05$; **, $P<0.01{ }^{* * *}, P<0.001$; ****, $\left.P<0.0001\right)$.

\section{Data Availability}

Microarray expression data are deposited in the Gene Expression Omnibus (GEO), accession number GSE141127. Raw single-cell RNA-seq data and corresponding UMI count matrices are deposited in GEO, accession number GSE141263. Raw bulk RNA-seq data and corresponding expression counts are deposited in GEO, accession number GSE141262.

\section{Authors' Disclosures}

J. Utikal reports personal fees and nonfinancial support from Amgen, Bristol Myers Squibb, GSK, Merck Sharp and Dohme, Novartis, Pierre Fabre, Roche, and Sanofi and grants from Apogenix, Noxxon Pharma, Elsalys Biotech, TILT Biotherapeutics, BioNTech RNA Pharmaceuticals, and RHEACELL outside the submitted work. S.R. Chintharlapalli reports personal fees from Eli Lilly and Company during the conduct of the study; personal fees and nonfinancial support from Eli Lilly and Company outside the submitted work. H.G. Augustin reports grants from Deutsche Forschungsgemeinschaft, grants from European Research Council, grants from Deutsche Forschungsgemeinschaft, grants from State of Baden-Wuertemberg, Germany, and grants from Deutsche Forschungsgemeinschaft during the conduct of the study. No disclosures were reported by the other authors.

\section{Authors' Contributions}

N. Gengenbacher: Conceptualization, data curation, formal analysis, supervision, investigation, visualization, methodology, writingoriginal draft, writing-review and editing. M. Singhal: Conceptualization, investigation, methodology, writing-review and editing. C. Mogler: Investigation. L. Hai: Investigation. L. Milde: Investigation. A.A. Abdul Pari: Investigation. E. Besemfelder: Investigation. C. Fricke: Investigation. D. Baumann: Investigation. S. Gehrs: Investigation. J. Utikal: Resources. M. Felcht: Resources. J. Hu: Investigation. M. Schlesner: Resources. R. Offringa: Resources. S.R. Chintharlapalli: Resources. H.G. Augustin: Conceptualization, resources, supervision, funding acquisition, writing-review and editing.

\section{Acknowledgments}

This work was supported by grants from the Deutsche Forschungsgemeinschaft [DFG; project C5 within CRC1366 "Vascular Control of Organ Function" (project number 39404578 to H.G. Augustin) and project C03 within CRC-TR209 "Liver Cancer - New mechanistic and therapeutic concepts in a solid tumor model" (project number 314905040 to H.G. Augustin)], the European Research Council
Advanced Grant "AngioMature" (project 787181 to H.G. Augustin), the DFG-funded Research Training Group 2099 "Hallmarks of Skin Cancer" (project P8 to M. Felcht and H.G. Augustin), and the State of Baden-Württemberg Foundation special program "Angioformatics Single Cell Platform." The authors would like to thank Prof. Dr. Kato (Chubu University) and Prof. Dr. Umansky (University Medical Center Mannheim) for providing MT-ret mice. We thank Prof. Dr. Bosenberg (Yale) for providing Tyr-Cre $e^{\mathrm{ERT} 2} \times \mathrm{Braf}^{\mathrm{\gamma} 600 \mathrm{E}} \times \mathrm{Pten}^{\mathrm{f} / \mathrm{f}} \times$ Ctnnb1 $1^{\text {Exon3 } \mathrm{Al} /+}$ mice. We thank Prof. Dr. Oliver (Northwestern Medicine) for providing Prox1-Cre ${ }^{\text {ERT2 }}$ mice. We thank Prof. Dr. Angel (DKFZ) for providing mTmG mice. We thank Prof. Dr. Sahagian for providing 4T1-Luc cells. We thank Johnny Eugene Croy (Eli Lilly and Company) for antibody production. We are most grateful for the excellent technical support of the Flow Cytometry, the Light Microscopy, the Small Animal Imaging, the Genomics and Proteomics, the Omics IT and Data Management, and the Laboratory Animal Facilities of the DKFZ.

\section{REFERENCES}

1. Dillekås H, Rogers MS, Straume O. Are $90 \%$ of deaths from cancer caused by metastases? Cancer Med 2019;8:5574-76.

2. Chitty JL, Filipe EC, Lucas MC, Herrmann D, Cox TR, Timpson P. Recent advances in understanding the complexities of metastasis. F1000Res 2018;7:1169.

3. Stacker SA, Williams SP, Karnezis T, Shayan R, Fox SB, Achen MG. Lymphangiogenesis and lymphatic vessel remodelling in cancer. Nat Rev Cancer 2014;14:159-72.

4. Dieterich LC, Detmar M. Tumor lymphangiogenesis and new drug development. Adv Drug Deliv Rev 2016;99:148-60.

5. He Y, Rajantie I, Pajusola K, Jeltsch M, Holopainen T, Yla-Herttuala $\mathrm{S}$, et al. Vascular endothelial cell growth factor receptor 3-mediated activation of lymphatic endothelium is crucial for tumor cell entry and spread via lymphatic vessels. Cancer Res 2005;65:4739-46.

6. Chen Y, Keskin D, Sugimoto H, Kanasaki K, Phillips PE, Bizarro L, et al. Podoplanin+ tumor lymphatics are rate limiting for breast cancer metastasis. PLoS Biol 2018;16:e2005907.

7. Gershenwald JE, Scolyer RA, Hess KR, Sondak VK, Long GV, Ross $\mathrm{MI}$, et al. Melanoma staging: evidence-based changes in the american joint committee on cancer eighth edition cancer staging manual. CA Cancer J Clin 2017;67:472-92.

8. Tas F, Erturk K. Histological lymphovascular invasion is associated with nodal involvement, recurrence, and survival in patients with cutaneous malignant melanoma. Int J Derm 2017;56:166-70.

9. Zhang S, Yi S, Zhang D, Gong M, Cai Y, Zou L. Intratumoral and peritumoral lymphatic vessel density both correlate with lymph node metastasis in breast cancer. Sci Rep 2017;7:40364.

10. Naxerova K, Reiter JG, Brachtel E, Lennerz JK, Van De Wetering $\mathrm{M}$, Rowan A, et al. Origins of lymphatic and distant metastases in human colorectal cancer. Science 2017;357:55-60.

11. Brown M, Assen FP, Leithner A, Abe J, Schachner H, Asfour G, et al. Lymph node blood vessels provide exit routes for metastatic tumor cell dissemination in mice. Science 2018;359:1408-11.

12. Pereira ER, Kedrin D, Seano G, Gautier O, Meijer EFJ, Jones D, et al. Lymph node metastases can invade local blood vessels, exit the node, and colonize distant organs in mice. Science 2018;359:1403-7.

13. Faries MB, Thompson JF, Cochran AJ, Andtbacka RH, Mozzillo N, Zager JS, et al. Completion dissection or observation for sentinelnode metastasis in melanoma. N Engl J Med 2017;376:2211-22. 
14. Galimberti V, Cole BF, Viale G, Veronesi P, Vicini E, Intra M, et al. Axillary dissection versus no axillary dissection in patients with breast cancer and sentinel-node micrometastases (IBCSG 23-01): 10-year follow-up of a randomised, controlled phase 3 trial. Lancet Oncol 2018;19:1385-93.

15. Gengenbacher N, Singhal M, Augustin HG. Preclinical mouse solid tumour models: status quo, challenges and perspectives. Nat Rev Cancer 2017; 17:751-65

16. Kerbel RS. A decade of experience in developing preclinical models of advanced- or early-stage spontaneous metastasis to study antiangiogenic drugs, metronomic chemotherapy, and the tumor microenvironment. Cancer J 2015;21:274-83.

17. Padera TP. Lymphatic metastasis in the absence of functional intratumor lymphatics. Science 2002;296:1883-6.

18. Padera TP, Stoll BR, Tooredman JB, Capen D, Tomaso ED, Jain RK. Cancer cells compress intratumour vessels. Nature 2004;427:695

19. Fidler IJ, Nicolson GL. Organ selectivity for implantation survival and growth of B16 melanoma variant tumor lines. J Natl Cancer Inst 1976;57:1199-202.

20. Kersten K, De Visser KE, Van Miltenburg MH, Jonkers J. Genetically engineered mouse models in oncology research and cancer medicine. EMBO Mol Med 2017;9:137-53.

21. Singh M, Lima A, Molina R, Hamilton P, Clermont AC, Devasthali V, et al. Assessing therapeutic responses in Kras mutant cancers using genetically engineered mouse models. Nat Biotechnol 2010;28: 585-93.

22. Eklund L, Kangas J, Saharinen P. Angiopoietin-Tie signalling in the cardiovascular and lymphatic systems. Clin Sci 2016;131:87-103.

23. Dellinger M, Hunter R, Bernas M, Gale N, Yancopoulos G, Erickson $\mathrm{R}$, et al. Defective remodeling and maturation of the lymphatic vasculature in Angiopoietin-2 deficient mice. Dev Biol 2008;319:309-20.

24. Gale NW, Thurston G, Hackett SF, Renard R, Wang Q, McClain $\mathrm{J}$, et al. Angiopoietin-2 is required for postnatal angiogenesis and lymphatic patterning, and only the latter role is rescued by angiopoietin-1. Dev Cell 2002;3:411-23.

25. Saharinen P, Eklund L, Alitalo K. Therapeutic targeting of the angiopoietin-TIE pathway. Nat Rev Drug Discov 2017;16:635-61.

26. Helfrich I, Edler L, Sucker A, Thomas M, Christian S, Schadendorf $\mathrm{D}$, et al. Angiopoietin-2 levels are associated with disease progression in metastatic malignant melanoma. Clin Cancer Res 2009;15: 1384-92.

27. Holopainen T, Saharinen P, D'Amico G, Lampinen A, Eklund L, Sormunen R, et al. Effects of angiopoietin-2-blocking antibody on endothelial cell-cell junctions and lung metastasis. J Natl Cancer Inst 2012;104:461-75.

28. Srivastava K, Hu J, Korn C, Savant S, Teichert M, Kapel SS, et al. Postsurgical adjuvant tumor therapy by combining anti-angiopoietin-2 and metronomic chemotherapy limits metastatic growth. Cancer Cell 2014;26:880-95

29. Park J-S, Kim I-K, Han S, Park I, Kim C, Bae J, et al. Normalization of tumor vessels by Tie2 activation and Ang2 inhibition enhances drug delivery and produces a favorable tumor microenvironment. Cancer Cell 2016;30:953-67.

30. Wu FTH, Man S, Xu P, Chow A, Paez-Ribes M, Lee CR, et al. Efficacy of cotargeting angiopoietin-2 and the VEGF pathway in the adjuvant postsurgical setting for early breast, colorectal, and renal cancers. Cancer Res 2016;76:6988-7000.

31. Monk BJ, Poveda A, Vergote I, Raspagliesi F, Fujiwara K, Bae D-S, et al. Final results of a phase 3 study of trebananib plus weekly paclitaxel in recurrent ovarian cancer (TRINOVA-1): long-term survival, impact of ascites, and progression-free survival-2. Gynecol Oncol 2016;143:27-34.

32. Marth C, Vergote I, Scambia G, Oberaigner W, Clamp A, Berger R, et al. ENGOT-ov-6/TRINOVA-2: randomised, double-blind, phase 3 study of pegylated liposomal doxorubicin plus trebananib or placebo in women with recurrent partially platinum-sensitive or resistant ovarian cancer. Eur J Cancer 2017;70:111-21.

33. Bendell JC, Sauri T, Gracián AC, Alvarez R, López-López C, GarcíaAlfonso P, et al. The McCAVE trial: vanucizumab plus mFOLFOX-6 versus bevacizumab plus mFOLFOX-6 in patients with previously untreated metastatic colorectal carcinoma (mCRC). Oncologist 2019;0291:e451-e459.

34. Vergote I, Scambia G, O’Malley DM, Van Calster B, Park SY, Del Campo JM, et al. Trebananib or placebo plus carboplatin and paclitaxel as first-line treatment for advanced ovarian cancer (TRINOVA-3/ ENGOT-ov2/GOG-3001): a randomised, double-blind, phase 3 trial. Lancet Oncol 2019;20:862-76.

35. Kato M, Takahashi M, Akhand AA, Liu W, Dai Y, Shimizu S, et al. Transgenic mouse model for skin malignant melanoma. Oncogene 1998; $17: 1885-8$.

36. Dadras SS, Lange-Asschenfeldt B, Velasco P, Nguyen L, Vora A, Muzikansky A, et al. Tumor lymphangiogenesis predicts melanoma metastasis to sentinel lymph nodes. Mod Pathol 2005;18: $1232-42$

37. Lambrechts D, Wauters E, Boeckx B, Aibar S, Nittner D, Burton O, et al. Phenotype molding of stromal cells in the lung tumor microenvironment. Nat Med 2018;24:1277-89.

38. Tirosh I, Izar B, Prakadan SM, Wadsworth MH II, Treacy D, Trombetta JJ, et al. Dissecting the multicellular ecosystem of metastatic melanoma by single-cell RNA-seq. Science 2016;352:189-96.

39. Han S, Lee SJ, Kim KE, Lee HS, Oh N, Park I, et al. Amelioration of sepsis by TIE2 activation-induced vascular protection. Sci Transl Med 2016;8:335ra55.

40. Nasarre P, Thomas M, Kruse K, Helfrich I, Wolter V, Deppermann C, et al. Host-derived angiopoietin-2 affects early stages of tumor development and vessel maturation but is dispensable for later stages of tumor growth. Cancer Res 2009;69:1324-33.

41. Schmittnaegel M, Rigamonti N, Kadioglu E, Cassará A, Wyser Rmili C, Kiialainen A, et al. Dual angiopoietin-2 and VEGFA inhibition elicits antitumor immunity that is enhanced by PD-1 checkpoint blockade. Sci Transl Med 2017;9:eaak9670.

42. Aebischer D, Iolyeva M, Halin C. The inflammatory response of lymphatic endothelium. Angiogenesis 2014;17:383-93.

43. Müller A, Homey B, Soto H, Ge N, Catron D, Buchanan ME, et al. Involvement of chemokine receptors in breast cancer metastasis. Nature 2001;410:50-6.

44. Shi M, Chen D, Yang D, Liu X-Y. CCL21-CCR7 promotes the lymph node metastasis of esophageal squamous cell carcinoma by upregulating MUC1. J Exp Clin Cancer Res 2015;34:149.

45. Zhao Q, Eichten A, Parveen A, Adler C, Huang Y, Wang W, et al. Single-cell transcriptome analyses reveal endothelial cell heterogeneity in tumors and changes following antiangiogenic treatment. Cancer Res 2018;78:2370-82.

46. del Toro R, Prahst C, Mathivet T, Siegfried G, Kaminker JS, Larrivee $\mathrm{B}$, et al. Identification and functional analysis of endothelial tip cellenriched genes. Blood 2010;116:4025-33.

47. Savant S, La Porta S, Budnik A, Busch K, Hu J, Tisch N, et al. The orphan receptor Tie1 Controls angiogenesis and vascular remodeling by differentially regulating Tie 2 in tip and stalk cells. Cell Rep 2015;12:1761-73

48. Souma T, Thomson BR, Heinen S, Anna Carota I, Yamaguchi S, Onay $\mathrm{T}$, et al. Context-dependent functions of angiopoietin 2 are determined by the endothelial phosphatase VEPTP. Proc Natl Acad Sci U S A 2018;115:1298-303.

49. Winderlich M, Keller L, Cagna G, Broermann A, Kamenyeva O, Kiefer F, et al. VE-PTP controls blood vessel development by balancing Tie-2 activity. J Cell Biol 2009;185:657-71.

50. Vaahtomeri K, Karaman S, Makinen T, Alitalo K. Lymphangiogenesis guidance by paracrine and pericellular factors. Genes Dev 2017;31:1615-34.

51. Weijts BG, van Impel A, Schulte-Merker S, de Bruin A. Atypical E2fs control lymphangiogenesis through transcriptional regulation of Ccbe1 and Flt4. PLoS One 2013;8:e73693.

52. Hiller JG, Perry NJ, Poulogiannis G, Riedel B, Sloan EK. Perioperative events influence cancer recurrence risk after surgery. Nat Rev Clin Oncol 2018;15:205-18.

53. Sanborn JZ, Chung J, Purdom E, Wang NJ, Kakavand H, Wilmott JS, et al. Phylogenetic analyses of melanoma reveal complex patterns 
of metastatic dissemination. Proc Natl Acad Sci U S A 2015;112: 10995-1000.

54. Gundem G, Van Loo P, Kremeyer B, Alexandrov LB, Tubio JMC, Papaemmanuil E, et al. The evolutionary history of lethal metastatic prostate cancer. Nature 2015;520:353-7.

55. Ubellacker JM, Tasdogan A, Ramesh V, Shen B, Mitchell EC, MartinSandoval MS, et al. Lymph protects metastasizing melanoma cells from ferroptosis. Nature 2020;585:113-8.

56. Rofstad EK, Huang R, Galappathi K, Andersen LMK, Wegner CS, Hauge A, et al. Functional intratumoral lymphatics in patient-derived xenograft models of squamous cell carcinoma of the uterine cervix: implications for lymph node metastasis. Oncotarget 2016; 56986-97.

57. Planas-Paz L, Lammert E. Mechanosensing in developing lymphatic vessels. Adv Anat Embryol Cell Biol 2014;214:23-40.

58. Khan S, Taverna F, Rohlenova K, Treps L, Geldhof V, de Rooij L, et al. EndoDB: a database of endothelial cell transcriptomics data. Nucleic Acids Res 2019;47:D736-D44.

59. Dieterich LC, Kapaklikaya K, Cetintas T, Proulx ST, Commerford CD, Ikenberg K, et al. Transcriptional profiling of breast cancer-associated lymphatic vessels reveals VCAM- 1 as regulator of lymphatic invasion and permeability. Int J Cancer 2019;145:2804-15.

60. Clasper S, Royston D, Baban D, Cao Y, Ewers S, Butz S, et al. A novel gene expression profile in lymphatics associated with tumor growth and nodal metastasis. Cancer Res 2008;68:7293-303.

61. Goveia J, Rohlenova K, Taverna F, Treps L, Conradi LC, Pircher A, et al. An integrated gene expression landscape profiling approach to identify lung tumor endothelial cell heterogeneity and angiogenic candidates. Cancer Cell 2020;37:21-36.

62. Fujimoto N, He Y, D’Addio M, Tacconi C, Detmar M, Dieterich LC. Single-cell mapping reveals new markers and functions of lymphatic endothelial cells in lymph nodes. PLOS Biol 2020;18:e3000704.

63. Takeda A, Hollmén M, Dermadi D, Pan J, Brulois KF, Kaukonen $\mathrm{R}$, et al. Single-cell survey of human lymphatics unveils marked endothelial cell heterogeneity and mechanisms of homing for neutrophils. Immunity 2019;51:561-72.

64. Xiang M, Grosso R, Takeda A, Pan J, Bekkhus T, Bruloi K, et al. A single-cell transcriptional roadmap of the mouse and human lymph node lymphatic vasculature. Front Cardiovasc Med 2020;7:52.

65. Elyada E, Bolisetty M, Laise P, Flynn WF, Courtois ET, Burkhart RA, et al. Cross-species single-cell analysis of pancreatic ductal adenocarcinoma reveals antigen-presenting cancer-associated fibroblasts. Cancer Discov 2019;9:1102-23.

66. Lane RS, Femel J, Breazeale AP, Loo CP, Thibault G, Kaempf A, et al. IFN gamma-activated dermal lymphatic vessels inhibit cytotoxic T cells in melanoma and inflamed skin. J Exp Med 2018;215 3057-74

67. Berendam SJ, Koeppel AF, Godfrey NR, Rouhani SJ, Woods AN, Rodriguez $\mathrm{AB}$, et al. Comparative transcriptomic analysis identifies a range of immunologically related functional elaborations of lymph node associated lymphatic and blood endothelial cells. Front Immunol 2019;10:816.

68. Hirosue S, Vokali E, Raghavan VR, Rincon-Restrepo M, Lund AW, Corthesy-Henrioud P, et al. Steady-state antigen scavenging, crosspresentation, and CD8+ T cell priming: a new role for lymphatic endothelial cells. J Immunol 2014;192:5002-11.

69. Lund AW, Duraes FV, Hirosue S, Raghavan VR, Nembrini C, Thomas $\mathrm{SN}$, et al. VEGF-C promotes immune tolerance in B16 melanomas and cross-presentation of tumor antigen by lymph node lymphatics. Cell Rep 2012;1:191-9.

70. Tewalt EF, Cohen JN, Rouhani SJ, Guidi CJ, Qiao H, Fahl SP, et al. Lymphatic endothelial cells induce tolerance via PD-L1 and lack of costimulation leading to high-level PD-1 expression on CD8 T cells. Blood 2012;120:4772-82.

71. Shen B, Shang Z, Wang B, Zhang L, Zhou F, Li T, et al. Genetic dissection of Tie pathway in mouse lymphatic maturation and valve development. Arterioscler Thromb Vasc Biol 2014;34:1221-30.
72. Zheng W, Nurmi H, Appak S, Sabine A, Bovay E, Korhonen EA, et al. Angiopoietin 2 regulates the transformation and integrity of lymphatic endothelial cell junctions. Gene Dev 2014;28: 1592-603.

73. Kim M, Allen B, Korhonen EA, Nitschké M, Yang HW, Baluk P, et al. Opposing actions of angiopoietin-2 on Tie2 signaling and FOXO1 activation. J Clin Invest 2016;126:3511-25.

74. Yuan HT, Khankin EV, Karumanchi SA, Parikh SM. Angiopoietin 2 is a partial agonist/antagonist of Tie2 signaling in the endothelium. Mol Cell Biol 2009;29:2011-22.

75. Scholz A, Harter PN, Cremer S, Yalcin BH, Gurnik S, Yamaji M, et al. Endothelial cell-derived angiopoietin-2 is a therapeutic target in treatment-naive and bevacizumab-resistant glioblastoma. EMBO Mol Med 2016;8:39-57.

76. Wang X, Bullock AJ, Zhang L, Wei L, Yu D, Mahagaokar K, et al. The role of angiopoietins as potential therapeutic targets in renal cell carcinoma. Transl Oncol 2014;7:188-95.

77. Goede V, Coutelle O, Neuneier J, Reinacher-Schick A, Schnell R, Koslowsky TC, et al. Identification of serum angiopoietin-2 as a biomarker for clinical outcome of colorectal cancer patients treated with bevacizumab-containing therapy. Br J Cancer 2010;103: $1407-14$

78. Sfiligoi C, de Luca A, Cascone I, Sorbello V, Fuso L, Ponzone R, et al. Angiopoietin-2 expression in breast cancer correlates with lymph node invasion and short survival. Int J Cancer 2003;103: 466-74.

79. Kienast Y, Klein C, Scheuer W, Raemsch R, Lorenzon E, Bernicke D, et al. Ang-2-VEGF-A CrossMab, a novel bispecific human IgG1 antibody blocking VEGF-A and Ang-2 functions simultaneously, mediates potent antitumor, antiangiogenic, and antimetastatic efficacy. Clin Cancer Res 2013;19:6730-40.

80. Minami T, Jiang S, Schadler K, Suehiro J, Osawa T, Oike Y, et al. The calcineurin-NFAT-angiopoietin-2 signaling axis in lung endothelium is critical for the establishment of lung metastases. Cell Rep 2013;4:709-23.

81. Mazzieri R, Pucci F, Moi D, Zonari E, Ranghetti A, Berti A, et al. Targeting the ANG2/TIE2 axis inhibits tumor growth and metastasis by impairing angiogenesis and disabling rebounds of proangiogenic myeloid cells. Cancer Cell 2011;19:512-26.

82. Schulz P, Fischer C, Detjen KM, Rieke S, Hilfenhaus G, von Marschall $Z$, et al. Angiopoietin-2 drives lymphatic metastasis of pancreatic cancer. FASEB J 2011;25:3325-35.

83. Tvedskov TF, Jensen MB, Kroman N, Balslev E. Iatrogenic displacement of tumor cells to the sentinel node after surgical excision in primary breast cancer. Breast Cancer Res Treat 2012;131:223-9.

84. Gandalovičová A, Rosel D, Fernandes M, Veselý P, Heneberg P, Čermák V, et al. Migrastatics -anti-metastatic and anti-invasion drugs: promises and challenges. Trends Cancer 2017;3: 391-406.

85. Damsky WE, Curley DP, Santhanakrishnan M, Rosenbaum LE, Platt JT, Gould Rothberg BE, et al. $\beta$-Catenin signaling controls metastasis in Braf-activated Pten-deficient melanomas. Cancer Cell 2011;20:741-54.

86. Muzumdar MD, Tasic B, Miyamichi K, Li L, Luo L. A global doublefluorescent cre reporter mouse. Genesis 2007;45:593-605.

87. Teichert M, Milde L, Holm A, Stanicek L, Gengenbacher N, Savant S, et al. Pericyte-expressed Tie 2 controls angiogenesis and vessel maturation. Nat Commun 2017;8:16106.

88. Mathieu M, Labrecque N. Murine superficial lymph node surgery. J Vis $\operatorname{Exp} 2012 ; \mathrm{e} 3444$.

89. Wang X, Ma K, Yang Z, Cui J, He H, Hoffman AR, et al. Systematic correlation analyses of circulating tumor cells with clinical variables and tumor markers in lung cancer patients. J Cancer 2017;8: 3099-104.

90. Livak KJ, Schmittgen TD. Analysis of relative gene expression data using real-time quantitative PCR and the $2-\Delta \Delta C T$ method. Methods 2001;25:402-8. 
91. Stuart T, Butler A, Hoffman P, Hafemeister C, Papalexi E, Mauck WM, et al. Comprehensive integration of single-cell data. Cell 2019;177: 1888-902.

92. Butler A, Hoffman P, Smibert P, Papalexi E, Satija R. Integrating single-cell transcriptomic data across different conditions, technologies, and species. Nat Biotechnol 2018;36:411-20.

93. Reimand J, Arak T, Adler P, Kolberg L, Reisberg S, Peterson H, et al. $\mathrm{g}$ :Profiler - a web server for functional interpretation of gene lists (2016 update). Nucleic Acids Res 2016;44:W83-W9.
94. Dobin A, Davis CA, Schlesinger F, Drenkow J, Zaleski C, Jha S, et al. STAR: ultrafast universal RNA-seq aligner. Bioinformatics 2013;29:15-21.

95. Love MI, Huber W, Anders S. Moderated estimation of fold change and dispersion for RNA-seq data with DESeq2. Genome Biol 2014;15:550.

96. Subramanian A, Tamayo P, Mootha VK, Mukherjee S, Ebert BL, Gillette MA, et al. Gene set enrichment analysis: a knowledge-based approach for interpreting genome-wide expression profiles. Proc Natl Acad Sci U S A 2005;102:15545-50. 